\title{
Energy Scaling Laws for Distributed Inference in Random Fusion Networks
}

\author{
Animashree Anandkumar, Student Member, IEEE, Joseph E. Yukich, Lang Tong, Fellow, IEEE, and Ananthram \\ Swami, Fellow, IEEE
}

\begin{abstract}
The energy scaling laws of multihop data fusion networks for distributed inference are considered. The fusion network consists of randomly located sensors distributed i.i.d. according to a general spatial distribution in an expanding region. Under Markov random field (MRF) hypotheses, among the class of data-fusion policies which enable optimal statistical inference at the fusion center using all the sensor measurements, the policy with the minimum average energy consumption is bounded below by the average energy of fusion along the minimum spanning tree, and above by a suboptimal policy, referred to as Data Fusion for Markov Random Fields (DFMRF). Scaling laws are derived for the energy consumption of the optimal and suboptimal fusion policies. It is shown that the average asymptotic energy of the DFMRF scheme is strictly finite for a class of MRF models with Euclidean stabilizing dependency graphs.
\end{abstract}

Index Terms-Distributed inference, graphical models, Euclidean random graphs, stochastic geometry and data fusion.

\section{INTRODUCTION}

W E CONSIDER the problem of distributed statistical inference in a network of randomly located sensors taking measurements and transporting the locally processed data to a designated fusion center. The fusion center then makes an inference about the underlying phenomenon based on the data collected from all the sensors.

For statistical inference using wireless sensor networks, energy consumption is an important design parameter. The transmission power required to reach a receiver distance $d$ away with a certain signal-to-noise ratio (SNR) scales in the order of $d^{\nu}$, where $2 \leq \nu \leq 6$ is the path loss [3]. Therefore, the cost of moving data from sensor locations to the fusion

Manuscript received 25 August 2008; revised 1 February 2009. Parts of this paper were presented at [1], [2]. This work was supported in part through collaborative participation in Communications and Networks Consortium sponsored by the U. S. Army Research Laboratory under the Collaborative Technology Alliance Program, Cooperative Agreement DAAD19-01-2-0011 and by the Army Research Office under Grant ARO-W911NF-06-1-0346. The first author is supported by the IBM Ph.D Fellowship for the year 2008-09 and is currently a visiting student at MIT, Cambridge, MA 02139. The second author was partially supported by NSA grant H98230-06-1-0052 and NSF grant DMS-0805570. The U. S. Government is authorized to reproduce and distribute reprints for Government purposes notwithstanding any copyright notation thereon.

A. Anandkumar and L. Tong are with the School of Electrical and Computer Engineering, Cornell University, Ithaca, NY 14853, USA (e-mail: \{a332@,1tong@ece.\}cornell.edu).

J.E. Yukich is with the Department of Mathematics, Lehigh University, Bethlehem, Pa. 18015 (e-mail: joseph.yukich@lehigh.edu).

A. Swami is with the Army Research Laboratory, Adelphi, MD 20783 USA (e-mail: a.swami@ieee.org).

Digital Object Identifier 10.1109/JSAC.2009.090916. center, either through direct transmissions or through multihop forwarding, significantly affects the lifetime of the network.

\section{A. Scalable data fusion}

We investigate the cost of data fusion for inference, and its scaling behavior with the size of the network and the area of deployment. In particular, for a network of $n$ random sensors located at points $\mathbf{V}_{n}=\left\{V_{1}, \cdots, V_{n}\right\}$ in $\mathbb{R}^{2}$, a fusion policy $\pi_{n}$ maps $\mathbf{V}_{n}$ to a set of scheduled transmissions and computations. The average cost (e.g., energy) of a policy is given by

$$
\overline{\mathcal{E}}\left(\pi_{n}\left(\mathbf{V}_{n}\right)\right):=\frac{1}{n} \sum_{i \in \mathbf{V}_{n}} \mathcal{E}_{i}\left(\pi_{n}\left(\mathbf{V}_{n}\right)\right),
$$

where $\mathcal{E}_{i}\left(\pi_{n}\left(\mathbf{V}_{n}\right)\right)$ is the cost at node $i$ under policy $\pi_{n}$. The above average cost is random, and we are interested in its scalability in random networks as $n \rightarrow \infty$.

Definition 1 (Scalable Policy): A sequence of policies $\pi:=\left(\pi_{n}\right)_{n \geq 1}$ is scalable on average if

$$
\lim _{n \rightarrow \infty} \mathbb{E}\left(\overline{\mathcal{E}}\left(\pi_{n}\left(\mathbf{V}_{n}\right)\right)\right)=\overline{\mathcal{E}}_{\infty}(\pi)<\infty
$$

where the expectation $\mathbb{E}$ is with respect to the random locations $\mathbf{V}_{n}$, and $\overline{\mathcal{E}}_{\infty}(\pi)$ is referred to as the scaling constant. A sequence of policies $\pi_{n}$ is weakly scalable if

$$
\left.\mathrm{p} \lim _{n \rightarrow \infty} \overline{\mathcal{E}}\left(\pi\left(\mathbf{V}_{n}\right)\right)\right)=\overline{\mathcal{E}}_{\infty}(\pi)<\infty,
$$

where $\mathrm{p}$ lim denotes convergence in probability. It is strongly scalable if the above average energy converges almost surely and is $L^{2}$ (mean-squared) scalable if the convergence is in mean square.

Hence, a scalable fusion policy implies a finite average energy expenditure even as the network size increases. We focus mostly on the $L^{2}$ scalability of the fusion policies, which implies weak and average scalability [4]. Further, we are interested in lossless data-fusion policies which enable the fusion center to perform optimal statistical inference with the best inference accuracy as if all the raw sensor data were available.

To motivate this study, first consider two simple fusion policies: the direct transmission policy (DT) in which all sensors transmit directly to the fusion center (single hop), and the shortest-path (SP) policy, where each node forwards its raw data to the fusion center using the shortest-path route without any data combination at the intermediate nodes. 
We assume, for now, that $n$ sensor nodes are uniformly distributed in a square of area $n$. It is perhaps not surprising that neither of the above two policies is scalable as $n \rightarrow$ $\infty$. For the DT policy ${ }^{1}$, intuitively, the average transmission range from the sensors to the fusion center scales as $\sqrt{n}$, thus $\overline{\mathcal{E}}\left(\mathrm{DT}\left(\mathbf{V}_{n}\right)\right)$ scales as $n^{\frac{\nu}{2}}$. On the other hand, we expect the SP policy to have better scaling since it chooses the best multihop path to forward data from each node to the fusion center. However, even in this case, there is no finite scaling. Here, the average number of hops in the shortest path from a node to the fusion center scales in the order of $\sqrt{n}$, and thus, $\overline{\mathcal{E}}\left(\operatorname{SP}\left(\mathbf{V}_{n}\right)\right)$ scales in the order of $\sqrt{n}$. Rigorously establishing the scaling laws for these two non-scalable policies is not crucial at this point since the same scaling laws can be easily established for regular networks when sensor nodes are on two-dimensional lattice points. See [5].

Are there scalable policies for data fusion? Among all the fusion policies not performing data combination at the intermediate nodes, the shortest-path (SP) policy minimizes the total energy. Thus, no scalable policy exists unless nodes cooperatively combine their information, a process known as data aggregation. Data aggregation, however, must be considered in conjunction with the performance requirements of specific applications. In this paper, we assume that optimal statistical inference is performed at the fusion center as if all the raw sensor data were available, and this places a constraint on data aggregation. For instance, it rules out sub-sampling of the sensor field, considered in [6].

\section{B. Summary of results and contributions}

In this paper, we investigate the energy scaling laws of lossless fusion policies which are allowed to perform data aggregation at the intermediate nodes, but ensure that the fusion center achieves the same inference accuracy as if all the raw observations were collected without any data combination. We assume that the underlying binary hypotheses for the sensor measurements can be modeled as Markov random fields (MRF).

For sensor locations $\mathbf{V}_{n}$ and possibly correlated sensor measurements, finding the minimum energy fusion policy under the constraint of optimal inference is given by

$$
\mathcal{E}\left(\pi^{*}\left(\mathbf{V}_{n}\right)\right)=\inf _{\pi \in \mathfrak{A}} \sum_{i \in \mathbf{V}_{n}} \mathcal{E}_{i}\left(\pi\left(\mathbf{V}_{n}\right)\right)
$$

where $\mathfrak{A}$ is the set of valid lossless data-fusion policies

$\mathfrak{A}:=\{\pi:$ optimal inference is achieved at the fusion center $\}$.

In general, the above optimization is NP-hard [7], and hence, studying its energy scaling behavior directly is intractable. We establish upper and lower bounds on the energy of this optimal policy $\pi^{*}$ and analyze the scaling behavior of these bounds. The lower bound is obtained via a policy conducting fusion along the Euclidean minimum spanning tree (MST), which is shown to be optimal when the sensor measurements are statistically independent under both hypotheses. The upper bound on the optimal fusion policy is established through a

\footnotetext{
${ }^{1}$ The direct transmission policy may not even be feasible, depending on the maximum transmission power constraints at the sensors.
}

specific suboptimal fusion policy, referred to as Data Fusion over Markov Random Fields (DFMRF). DFMRF becomes optimal when observations are independent under either hypothesis, where it reduces to fusion along the MST. For certain spatial dependencies among sensor measurements of practical significance, such as the Euclidean 1-nearest neighbor graph, DFMRF has an approximation ratio 2, i.e., its energy is no more than twice that of the optimal fusion policy, independent of the size and configuration of the network.

We then proceed to establish a number of asymptotic properties of the DFMRF policy in Section IV, including its energy scalability, its performance bounds, and the approximation ratio with respect to the optimal fusion policy when the sensor measurements have dependencies described by a $k$-nearest neighbor graph or a disc graph (continuum percolation). Applying techniques developed in [8]-[11], we provide a precise characterization of the scaling bounds as a function of sensor density and sensor placement distribution. These asymptotic bounds for DFMRF, in turn, imply that the optimal fusion policy is also scalable. Hence, we use the DFMRF policy as a vehicle to establish scaling laws for optimal fusion. Additionally, we use the energy scaling constants to optimize the distribution of the sensor placements. For independent measurements conditioned on each hypothesis, we show that the uniform distribution of the sensor nodes minimizes the asymptotic average energy consumption over all i.i.d spatial placements when the path-loss exponent of transmission is greater than two $(\nu>2)$. For $\nu \in[0,2)$, we show that the uniform distribution is, in fact, the most expensive ${ }^{2}$ node configuration in terms of routing costs. We further show that the optimality of the uniform node distribution applies for both the lower and upper bounds on the average energy consumption of the optimal fusion policy under Markov random field measurements with $k$-nearest neighbor dependency graph or the disc dependency graph under certain conditions.

To the best of our knowledge, our results are the first to establish the energy scalability of data fusion for certain correlation structures of the sensor measurements. The use of energy scaling laws for the design of efficient sensor placement is new and has direct engineering implications. The fusion policy DFMRF first appeared in [12], and is made precise here with detailed asymptotic analysis using the weak law of large numbers (WLLN) for stabilizing Euclidean graph functionals. One should not expect that scalable data fusion is always possible, and at the end of Section IV, we discuss examples of correlation structures where scalable lossless datafusion policy does not exist.

\section{Prior and related work}

The seminal work of Gupta and Kumar [13] on the capacity of wireless networks has stimulated extensive studies covering a broad range of networking problems with different performance metrics. See also [14]. Here, we limit ourselves to the related works on energy consumption and data fusion for statistical inference.

Results on scaling laws for energy consumption are limited. In [15], energy scaling laws for multihop wireless networks

\footnotetext{
${ }^{2}$ The path-loss exponent for wireless transmissions satisfies $\nu>2$.
} 
(without any data fusion) are derived under different routing strategies. The issue of node placement for desirable energy scaling has been considered in [16], [17], where it is argued that uniform node placement, routinely considered in the literature, has poor energy performance when there is no data fusion. It is interesting to note that, for fusion networks, uniform sensor distribution is in fact optimal among a general class of distributions. See Section IV-B.

Energy-efficient data fusion has received a great deal of attention over the past decade. See a few recent surveys in [18], [19]. It has been recognized that sensor observations tend to be correlated, and that correlations should be exploited through data fusion. One line of approach is the use of distributed compression with the aim of reconstructing all the measurements at the fusion center. Examples of such approaches can be found in [20]-[22].

While sending data from all sensors to the fusion center is certainly sufficient to ensure optimal inference, it is not necessary. More relevant to our work is the idea of data aggregation, e.g., [23]-[25]. Finding aggregation policies for correlated data, however, is nontrivial; it depends on the specific applications for which the sensor network is designed. Perhaps a more precise notion of aggregation is in-network function computation where certain functions are computed by passing intermediate values among nodes [26]-[29]. However, these works are mostly concerned with computing symmetric functions such as the sum function, which in general, do not satisfy the constraint of optimal statistical inference at the fusion center.

In the context of statistical inference using wireless sensor networks, the idea of aggregation and in-network processing has been explored by several authors. See [30]-[36]. Most relevant to our work are [30]-[34] where the Markovian correlation structures of sensor measurements are exploited explicitly. These results mostly deal with one-dimensional node placements, and do not deal with randomly placed nodes or energy scaling laws.

The results presented in this paper extend some of our earlier work in the direction of scaling-law analysis in random fusion networks. In [7], [12], [37], for fixed network size and node placement, we analyzed the minimum energy fusion policy for optimal inference and showed that it reduces to the Steiner-tree optimization problem under certain constraints. We also proposed a suboptimal fusion policy called the $\mathrm{DFMRF}^{3}$. In [38], we analyzed the optimal sensor density for uniform node placement which maximizes the inference error exponent under an average energy constraint, and in [39], [40], we derived the error exponent for MRF hypotheses. In [6], we analyzed optimal sensor selection (i.e., sub-sampling) policies for achieving tradeoff between fusion costs and inference performance.

The energy scaling laws derived in this paper rely heavily on several results on the law of large numbers for geometric random graphs. We have extensively borrowed the formulations and techniques of Penrose and Yukich [11], [41]. See Appendix A for a brief description and [8], [9], [42] for detailed expositions of these ideas.

${ }^{3}$ The DFMRF policy is referred to as AggMST in [7], [37].

\section{SySTEM MODEL}

In this paper, we consider various graphs. Chief among these are (i) dependency graphs specifying the correlation structure of sensor measurements, (ii) network graphs denoting the (directed) set of feasible links for communication, and (iii) fusion policy digraphs denoting the (directed) links used by a policy to route and aggregate data according to a given sequence. Note that the fusion policy takes the dependency graph and the network graph as inputs and outputs the fusionpolicy digraph. The dependency and network graphs can be independently specified and are in general, functions of the sensor locations.

\section{A. Stochastic model of sensor locations}

We assume that $n$ sensor nodes (including the fusion center) are placed randomly with sensor $i$ located at $V_{i} \in \mathbb{R}^{2}$. By convention, the fusion center is denoted by $i=1$, and is located at $V_{1} \in \mathbb{R}^{2}$. We denote the set of locations of the $n$ sensors by $\mathbf{V}_{n}:=\left\{V_{1}, \ldots, V_{n}\right\}$. For our scaling law analysis, we consider a sequence of sensor populations placed in expanding square regions $Q_{\frac{n}{\lambda}}$ of area $\frac{n}{\lambda}$ and centered at the origin $\mathbf{0} \in \mathbb{R}^{2}$, where we fix $\lambda$ as the overall sensor density and let the number of sensors $n \rightarrow \infty$.

To generate sensor locations $V_{i}$, first let $Q_{1}:=\left[-\frac{1}{2}, \frac{1}{2}\right]^{2}$ be the unit-area square ${ }^{4}$, and $X_{i} \stackrel{i . i . d .}{\sim} \tau, 1 \leq i \leq n$, be a set of $n$ independent and identically distributed (i.i.d.) random variables distributed on support $Q_{1}$ according to $\tau$. Here, $\tau$ is a probability density function (pdf) on $Q_{1}$ which is bounded away from zero and infinity. We then generate $V_{i}$ by scaling $X_{i}$ accordingly: $V_{i}=\sqrt{\frac{n}{\lambda}} X_{i} \in Q_{\frac{n}{\lambda}}$. A useful special case is the uniform distribution $(\tau \equiv 1)$. Let $\mathcal{P}_{a}$ be the homogeneous Poisson distribution on $\mathbb{R}^{2}$ with intensity $a>0$.

\section{B. Graphical inference model: dependency graphs}

We consider the statistical inference problem of simple binary hypothesis testing, $\mathcal{H}_{0}$ vs. $\mathcal{H}_{1}$, on a pair of Markov random fields. Under regularity conditions [43], a MRF is defined by its (undirected) dependency graph $\mathcal{G}$ and an associated pdf $f(\cdot \mid \mathcal{G})$.

Under hypothesis $\mathcal{H}_{k}$ and sensor location set $\mathbf{V}_{n}=$ $\left\{V_{1}, \cdots, V_{n}\right\}$ generated according to the stochastic model in Section II-A, we assume that the dependency graph $\mathcal{G}_{k}:=$ $\left(\mathbf{V}_{n}, E_{k}\right)$ models the correlation among the sensor observations. Note that the node location set $\mathbf{V}_{n}$ under the two hypotheses are identical. Set $E_{k}$ is the set of edges of the dependency graph $\mathcal{G}_{k}$, and it defines the correlations of the sensor observations, as described in the next section.

We restrict our attention to proximity-based Euclidean dependency graphs. In particular, we consider two classes of dependency graphs ${ }^{5}$ : the (undirected) $k$-nearest neighbor graph $(k-\mathrm{NNG})$ and the disc graph, also known as the continuum percolation graph. We expect that our results extend to other

\footnotetext{
${ }^{4}$ The results in this paper hold for $\tau$ defined on any convex unit area.

${ }^{5}$ The $k$-nearest neighbor graph $(k$-NNG) has edges $(i, j)$ if $i$ is one of the top $k$ nearest neighbors of $j$ or viceversa, and ties are arbitrarily broken. The disc graph has edges between any two points within a certain specified Euclidean distance (radius).
} 
locally-defined dependency structures such as the Delaunay, Voronoi, the minimum spanning tree, the sphere of influence and the Gabriel graphs. An important property of the aforementioned graphs is a certain stabilization property (discussed in Appendix A) facilitating asymptotic scaling analysis.

\section{Graphical inference model: likelihood functions}

We denote the measurements from all the $n$ sensors placed at fixed locations $\mathbf{v}_{n}$ by $\mathbf{Y}_{\mathbf{v}_{n}}$. The statistical inference problem can now be stated as the following hypothesis test:

$$
\begin{aligned}
& \mathcal{H}_{0}:\left[\mathbf{Y}_{\mathbf{V}_{n}}, \mathbf{V}_{n}\right] \sim f\left(\mathbf{y}_{\mathbf{v}_{n}} \mid \mathcal{G}_{0}\left(\mathbf{v}_{n}\right), \mathcal{H}_{0}\right) \prod_{i=1}^{n} \tau\left(\sqrt{\frac{\lambda}{n}} v_{i}\right), \\
& \mathcal{H}_{1}:\left[\mathbf{Y}_{\mathbf{V}_{n}}, \mathbf{V}_{n}\right] \sim f\left(\mathbf{y}_{\mathbf{v}_{n}} \mid \mathcal{G}_{1}\left(\mathbf{v}_{n}\right), \mathcal{H}_{1}\right) \prod_{i=1}^{n} \tau\left(\sqrt{\frac{\lambda}{n}} v_{i}\right),
\end{aligned}
$$

where $f\left(\mathbf{y}_{\mathbf{v}_{n}} \mid \mathcal{G}_{k}, \mathcal{H}_{k}\right)$ is the pdf of $\mathbf{Y}_{\mathbf{v}_{n}}$ given the dependency graph $\mathcal{G}_{k}\left(\mathbf{v}_{n}\right)$ under hypothesis $\mathcal{H}_{k}$. Note that the sensor locations $\mathbf{V}_{n}$ have the same distribution under either hypothesis. Therefore, only the conditional distribution of $\mathbf{Y}_{\mathbf{v}_{n}}$ given the sensor locations $\mathbf{V}_{n}=\mathbf{v}_{n}$ under each hypothesis is relevant for inference.

Under each hypothesis, the dependency graph specifies conditional-independence relations between the sensor measurements [43]

$$
Y_{i} \Perp \mathbf{Y}_{\mathbf{V}_{n} \backslash \mathcal{N}\left(i ; \mathcal{G}_{k}\right)} \mid\left\{\mathbf{Y}_{\mathcal{N}\left(i ; \mathcal{G}_{k}\right)}, \mathbf{V}_{n}\right\}, \quad \text { under } \mathcal{H}_{k},
$$

where $\mathcal{N}\left(i ; \mathcal{G}_{k}\right)$ is the set of neighbors of $i$ in $\mathcal{G}_{k}$, and $\Perp$ denotes conditional independence. In words, the measurement at a node is conditionally independent of the rest of the network, given the node locations $\mathbf{V}_{n}$ and the measurements at its neighbors in the dependency graph.

The celebrated Hammersley-Clifford theorem [44] states that, under the positivity condition ${ }^{6}$, the log-likelihood function of a MRF with dependency graph $\mathcal{G}_{k}$ can be expressed as

$$
-\log f\left(\mathbf{y}_{\mathbf{v}_{n}} \mid \mathcal{G}_{k}\left(\mathbf{v}_{n}\right), \mathcal{H}_{k}\right)=\sum_{c \in \mathfrak{C}_{k}} \psi_{k, c}\left(\mathbf{y}_{c}\right), \quad k=0,1
$$

where $\mathcal{C}_{k}$ is a collection of (maximal) cliques ${ }^{7}$ in $\mathcal{G}_{k}\left(\mathbf{v}_{n}\right)$, the functions $\psi_{k, c}$, known as clique potentials, are real valued, and not zero everywhere on the support of the distribution of $\mathbf{y}_{c}$.

We assume that the normalization constant (partition function) is already incorporated in the potential functions to ensure that (5) indeed describes a probability measure. In general, it is NP-hard to evaluate the normalization constant given arbitrary potential functions [45], but can be carried out at the fusion center without any need for communication of sensor measurements.

\footnotetext{
${ }^{6}$ The positivity condition rules out degeneracy among a subset of nodes: $Y_{1}=Y_{2} \ldots=Y_{k}$, where it is not required for every node to transmit at least once for computation of likelihood ratio.

${ }^{7} \mathrm{~A}$ clique is a complete subgraph, and a maximal clique is a clique which is not contained in a bigger clique.
}

\section{Communication model and energy consumption}

The set of feasible communication links form the (directed) network graph denoted by $\mathcal{N}_{g}\left(\mathbf{v}_{n}\right)$, for a given realization of sensor locations $\mathbf{V}_{n}=\mathbf{v}_{n}$. We assume that it is connected but not necessarily fully connected, and that it contains the Euclidean minimum spanning tree over the node set $\mathbf{v}_{n}$ and directed towards the fusion center $v_{1}$, denoted by $\operatorname{DMST}\left(\mathbf{v}_{n} ; v_{1}\right)$. Usually in the literature, in order to incorporate the maximum power constraints at the nodes, the network graph is assumed to be a disc graph with radius above the connectivity threshold [14], but we do not limit to this model. Transmissions are scheduled so as to not interfere with one other. Nodes are capable of adjusting their transmission power depending on the location of the receiver.

A fusion policy $\pi\left(\mathbf{v}_{n}\right)$ consists of a transmission schedule with the transmitter-receiver pairs and the aggregation algorithm that allows a node to combine its own and received values to produce a new communicating value. We model a fusion policy $\pi$ by a fusion-policy digraph, $\mathcal{F}_{\pi}:=\left(\mathbf{v}_{n}, \vec{E}_{\pi}\right)$, and $\vec{E}_{\pi}$ contains directed links. A directed ${ }^{8}$ link $\langle i, j\rangle$ denotes a direct transmission from $i$ to $j$ and is required to be a member in the network graph $\mathcal{N}_{g}\left(\mathbf{v}_{n}\right)$ for transmissions to be feasible. If one node communicates with another node $k$ times, $k$ direct links are present between these two nodes in the edge set $\vec{E}_{\pi}$ of the fusion policy $\pi$. Since we are only interested in characterizing the overall energy expenditure, the order of transmissions is not important; we only need to consider the associated cost with each link in $\vec{E}_{\pi}$ and calculate the sum cost for $\pi$.

Nodes communicate in the form of packets. Each packet contains bits for at most one (quantized) real variable and other overhead bits independent of the network size. We assume that all real variables ${ }^{9}$ are quantized to $K$ bits, and $K$ is independent of network size and is sufficiently large that quantization errors can be ignored. Thus, for node $i$ to transmit data to node $j$ which is distance $|i, j|$ away, we assume that node $i$ spends energy ${ }^{10} \gamma|i, j|^{\nu}$. Without loss of generality, we assume $\gamma=1$. Hence, given a fusion policy $\mathcal{F}_{\pi}=\left(\mathbf{v}_{n}, \vec{E}_{\pi}\right)$ of network size $n$, the average energy consumption is given by

$$
\overline{\mathcal{E}}\left(\pi\left(\mathbf{v}_{n}\right)\right)=\frac{1}{n} \mathcal{E}\left(\pi\left(\mathbf{v}_{n}\right)\right)=\frac{1}{n} \sum_{\langle i, j\rangle \in \vec{E}_{\pi}}|i, j|^{\nu}, \quad 2 \leq \nu \leq 6 .
$$

The model specification is now complete.

\section{Minimum Energy Data Fusion}

In this section, we present data-fusion policies aimed at minimizing energy expenditure under the constraint of optimal statistical inference at the fusion center, given in (2). The scalability of these policies is deferred to Section IV.

\footnotetext{
${ }^{8}$ We denote a directed link by $\langle i, j\rangle$ and an undirected link by $(i, j)$.

${ }^{9}$ In principle, the raw and aggregated data may require different amount of energy for communication, and can be incorporated into our framework.

${ }^{10}$ Since nodes only communicate a finite number of bits, we use energy instead of power as the cost measure.
} 


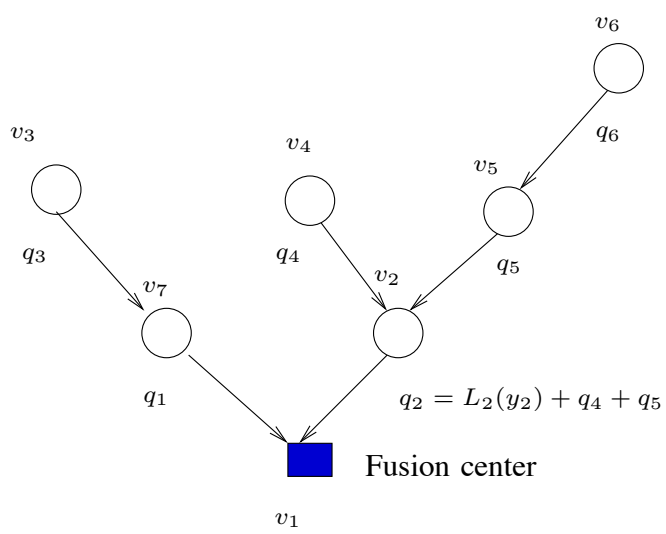

Fig. 1. The optimal fusion graph DMST for independent observations.

\section{A. Optimal data fusion: a reformulation}

We consider correlated sensor measurements under the Markov random field model. The inference problem, defined in (3), involves two different graphical models, each with its own dependency graph and associated likelihood function. They do share the same node location set $\mathbf{V}_{n}$ which allows us to merge the two graphical models into one.

For a given realization of sensor locations $\mathbf{V}_{n}=\mathbf{v}_{n}$, define the joint dependency graph $\mathcal{G}:=\left(\mathbf{v}_{n}, E\right)$, where $E:=E_{0} \bigcup E_{1}$, as the union of the two (random) dependency graphs $\mathcal{G}_{0}$ and $\mathcal{G}_{1}$. The minimal sufficient statistic ${ }^{11}$ is given by the loglikelihood ratio (LLR) [47]. With the substitution of (5), it is given by

$$
\begin{aligned}
L_{\mathcal{G}}\left(\mathbf{y}_{\mathbf{v}_{n}}\right) & :=\log \frac{f\left(\mathbf{y}_{\mathbf{v}_{n}} \mid \mathcal{G}_{0}\left(\mathbf{v}_{n}\right), \mathcal{H}_{0}\right)}{f\left(\mathbf{y}_{\mathbf{v}_{n}} \mid \mathcal{G}_{1}\left(\mathbf{v}_{n}\right), \mathcal{H}_{1}\right)} \\
& =\sum_{a \in \mathfrak{C}_{1}} \psi_{1, a}\left(\mathbf{y}_{a}\right)-\sum_{b \in \mathcal{C}_{0}} \psi_{0, b}\left(\mathbf{y}_{b}\right) \\
& :=\sum_{c \in \mathcal{C}} \phi_{c}\left(\mathbf{y}_{c}\right), \quad \mathcal{C}:=\mathcal{C}_{0} \bigcup \mathcal{C}_{1},
\end{aligned}
$$

where $\mathcal{C}$ is the set of maximal cliques in $\mathcal{G}$ and the effective potential functions $\phi_{c}$ are given by

$$
\phi_{c}\left(\mathbf{y}_{c}\right):=\sum_{a \in \mathfrak{C}_{1}, a \subset c} \psi_{1, a}\left(\mathbf{y}_{a}\right)-\sum_{b \in \mathfrak{C}_{0}, b \subset c} \psi_{0, b}\left(\mathbf{y}_{b}\right), \quad \forall c \in \mathcal{C} .
$$

Hereafter, we work with $\left(\mathcal{G}, L_{\mathcal{G}}\left(\mathbf{y}_{\mathbf{v}_{n}}\right)\right)$ and refer to the joint dependency graph $\mathcal{G}$ as just the dependency graph.

Note that the log-likelihood ratio is minimally sufficient [47] (i.e., maximum dimensionality reduction) implying maximum possible savings in routing energy through aggregation under the constraint of optimal statistical inference. Given a fixed node-location set $\mathbf{v}_{n}$, we can now reformulate the optimal data-fusion problem in (2) as the following optimization

$$
\mathcal{E}\left(\pi^{*}\left(\mathbf{v}_{n}\right)\right)=\inf _{\pi \in \widetilde{F}_{g}} \sum_{i \in \mathbf{v}_{n}} \mathcal{E}_{i}\left(\pi\left(\mathbf{v}_{n}\right)\right),
$$

\footnotetext{
${ }^{11}$ A sufficient statistic is a well-behaved function of the data, which is as informative as the raw data for inference. It is minimal if it is a function of every other sufficient statistic [46].
}

where $\mathfrak{F}_{\mathcal{G}}$ is the set of valid data-fusion policies

$$
\mathfrak{F}_{\mathcal{G}}:=\left\{\pi: L_{\mathcal{G}}\left(\mathbf{y}_{\mathbf{v}_{n}}\right) \text { computable at the fusion center }\right\} .
$$

Note that the optimization in (9) is a function of the dependency graph $\mathcal{G}\left(\mathbf{v}_{n}\right)$, and that the optimal solution is attained by some policy. In general, the above optimization is NP-hard [7].

\section{B. Minimum energy data fusion: a lower bound}

The following theorem gives a lower bound on the minimum energy in (9), given the joint dependency graph $\mathcal{G}$ and the pathloss exponent $\nu$. Let $\operatorname{MST}\left(\mathbf{v}_{n}\right)$ be the Euclidean minimum spanning tree over a realization of sensor locations $\mathbf{V}_{n}=\mathbf{v}_{n}$.

Theorem 1 (Lower bound on minimum energy expenditure): The following results hold:

1) the energy cost for the optimal fusion policy $\pi^{*}$ in (9) satisfies

$$
\mathcal{E}\left(\pi^{*}\left(\mathbf{v}_{n}\right)\right) \geq \mathcal{E}\left(\operatorname{MST}\left(\mathbf{v}_{n}\right)\right):=\sum_{e \in \operatorname{MST}\left(\mathbf{v}_{n}\right)}|e|^{\nu},
$$

2) the lower bound (10) is achieved (i.e., equality holds) when the observations are independent under both hypotheses. In this case, the optimal fusion policy $\pi^{*}$ aggregates data along $\operatorname{DMST}\left(\mathbf{v}_{n} ; v_{1}\right)$, the directed minimum spanning tree, with all the edges directed toward the fusion center $v_{1}$. Hence, the optimal fusion digraph $\mathcal{F}_{\pi^{*}}$ is the $\operatorname{DMST}\left(\mathbf{v}_{n} ; v_{1}\right)$.

Proof: We first prove part 2), for which we consider the case when observations are independent, and the log-likelihood ratio is given by

$$
L_{\mathcal{G}}\left(\mathbf{y}_{\mathbf{v}_{n}}\right)=\sum_{i \in \mathbf{v}_{n}} L_{i}\left(y_{i}\right), \quad L_{i}\left(y_{i}\right):=\log \frac{f_{1, i}\left(y_{i}\right)}{f_{0, i}\left(y_{i}\right)},
$$

where $f_{k, i}$ is the marginal pdf at node $i$ under $\mathcal{H}_{k}$. Consider $\operatorname{MST}\left(\mathbf{v}_{n}\right)$, whose links minimize $\sum_{e \in \operatorname{Tree}\left(\mathbf{v}_{n}\right)}|e|^{\nu}$. It is easy to check that at the fusion center, the log-likelihood ratio can be computed using the following aggregation policy along the $\operatorname{DMST}\left(\mathbf{v}_{n} ; v_{1}\right)$ as illustrated in Fig.1: each node $i$ computes the aggregated variable $q_{i}\left(\mathbf{y}_{\mathbf{v}_{n}}\right)$ from its predecessor and sends it to its immediate successor. The variable $q_{i}$ is given by the summation

$$
q_{i}\left(\mathbf{y}_{\mathbf{v}_{n}}\right):=\sum_{j \in \mathcal{N}_{p}(i)} q_{j}\left(\mathbf{y}_{\mathbf{v}_{n}}\right)+L_{i}\left(y_{i}\right),
$$

where $\mathcal{N}_{p}(i)$ is the set of immediate predecessors of $i$ in $\operatorname{DMST}\left(\mathbf{v}_{n} ; v_{1}\right)$.

To show part 1), we note that any data-fusion policy must have each node transmit at least once and that the transmission must ultimately reach the fusion center. This implies that the fusion digraph must be connected with the fusion center and the DMST with edge-weight $|e|^{\nu}$ minimizes the total energy under the above constraints. Hence, we have (10).

Note that the above lower bound in (10) is achievable when the measurements are independent under both hypotheses. It is interesting to note that data correlations, in general, increase the energy consumption under the constraint of optimal 


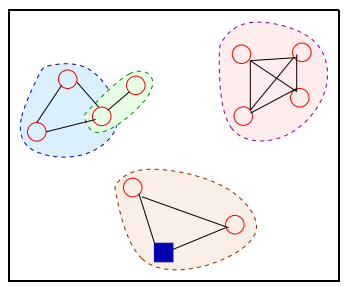

(a) Maximal cliques of dependency graph

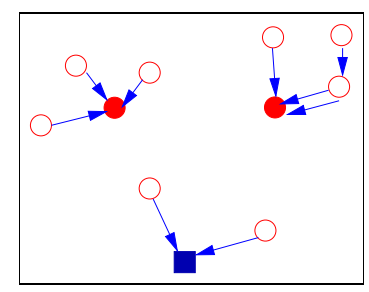

(b) Forwarding subgraph computes clique potentials

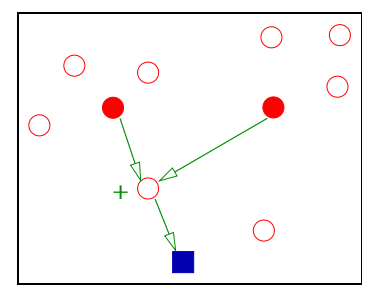

(c) Aggregation subgraph adds computed potentials

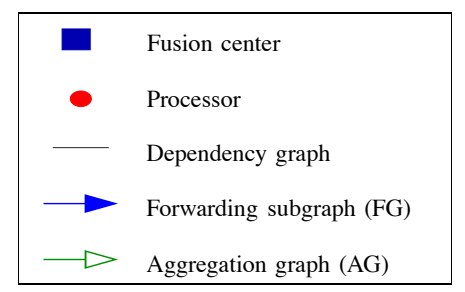

(d) Legend

Fig. 2. Schematic of dependency graph of Markov random field and stages of data fusion.

inference performance since the log-likelihood ratio in (7) cannot be decomposed fully in terms of the individual node measurements.

\section{Minimum energy data fusion: an upper bound}

We now devise a suboptimal data-fusion policy which gives an upper bound on the optimal energy in (9) for any given dependency graph $\mathcal{G}$ of the inference model. The suboptimal policy is referred to as Data Fusion on Markov Random Fields (DFMRF). It is a natural generalization of the MST aggregation policy, described in Theorem 1, which is valid only for independent measurements.

We shall use Fig. 2 to illustrate the idea behind DFMRF. Recalling that the log-likelihood ratio for hypothesis testing of Markov random fields is given by (7), DFMRF consists of two phases:

1) In the data forwarding phase, for each clique $c$ in the set of maximal cliques $\mathcal{C}$ of the dependency graph $\mathcal{G}$, a processor, denoted by $\operatorname{Proc}(c)$, is chosen arbitrarily amongst the members of the clique $c$. Each node in clique $c$ (other than the processor itself) forwards its raw data to $\operatorname{Proc}(c)$ via the shortest path using links in the network graph $\mathcal{N}_{g}$. The processor $\operatorname{Proc}(c)$ computes the clique-potential function $\phi_{c}\left(\mathbf{y}_{c}\right)$ using the forwarded data.

2) In the data-aggregation phase, processors compute the sum of the clique potentials along $\operatorname{DMST}\left(\mathbf{v}_{n} ; v_{1}\right)$, the directed MST towards the fusion center, thereby delivering the log-likelihood ratio in (7) to the fusion center.

Hence, the fusion-policy digraph for DFMRF is the union of the subgraphs in the above two stages, viz., forwarding subgraph $\left(\mathrm{FG}\left(\mathbf{v}_{n}\right)\right)$ and aggregation subgraph $\left(\mathrm{AG}\left(\mathbf{v}_{n}\right)\right)$. The total energy consumption of DFMRF is the sum of energies of the two subgraphs, given by

$$
\begin{aligned}
\mathcal{E}\left(\operatorname{DFMRF}\left(\mathbf{v}_{n}\right)\right)= & \sum_{c \in \mathcal{C}\left(\mathbf{v}_{n}\right)} \sum_{i \subset c} \mathcal{E}^{\mathrm{SP}}\left(i, \operatorname{Proc}(c) ; \mathcal{N}_{g}\right) \\
& +\mathcal{E}\left(\operatorname{MST}\left(\mathbf{v}_{n}\right)\right),
\end{aligned}
$$

where $\mathcal{E}^{\mathrm{SP}}\left(i, j ; \mathcal{N}_{g}\right)$ denotes the energy consumption for the shortest path from $i$ to $j$ using the links in the network graph $\mathcal{N}_{g}\left(\mathbf{v}_{n}\right)$ (set of feasible links for direct transmission). Recall that the network graph $\mathcal{N}_{g}$ is different from the dependency graph $\mathcal{G}$ since the former deals with communication while the latter deals with data correlation.
For independent measurements under either hypothesis, the maximal clique set $\mathcal{C}$ is trivially the set of vertices $\mathbf{v}_{n}$ itself and hence, DFMRF reduces to aggregation along the $\operatorname{DMST}\left(\mathbf{v}_{n} ; v_{1}\right)$, which is the optimal policy $\pi^{*}$ for independent observations. However, in general, DFMRF is not optimal. When the dependency graph $\mathcal{G}$ in (7) is the Euclidean 1-nearest neighbor graph, we now show that the DFMRF has a constant approximation ratio with respect to the optimal datafusion policy $\pi^{*}$ in (9) for any arbitrary node placement.

Theorem 2 (Approximation under 1-NNG dependency [12]): DFMRF is a 2-approximation fusion policy when the dependency graph $\mathcal{G}$ is the Euclidean 1-nearest neighbor graph for any fixed node set $\mathbf{v}_{n} \in \mathbb{R}^{2}$

$$
\frac{\mathcal{E}\left(\operatorname{DFMRF}\left(\mathbf{v}_{n}\right)\right)}{\mathcal{E}\left(\pi^{*}\left(\mathbf{v}_{n}\right)\right)} \leq 2 .
$$

Proof: Since 1-NNG is acyclic, the maximum clique size is 2. Hence, for DFMRF, the forwarding subgraph (FG) is the 1-NNG with arbitrary directions on the edges. We have

$$
\mathcal{E}\left(\mathrm{FG}\left(\mathbf{v}_{n}\right)\right)=\mathcal{E}\left(1-\mathrm{NNG}\left(\mathbf{v}_{n}\right)\right) \leq \mathcal{E}\left(\operatorname{MST}\left(\mathbf{v}_{n}\right)\right) .
$$

Thus,

$$
\begin{array}{rl}
\mathcal{E}\left(\operatorname{DFMRF}\left(\mathbf{v}_{n}\right)\right)= & \mathcal{E}\left(\mathrm{FG}\left(\mathbf{v}_{n}\right)\right)+\mathcal{E}\left(\operatorname{AG}\left(\mathbf{v}_{n}\right)\right), \\
\leq 2 & \mathcal{E}\left(\operatorname{MST}\left(\mathbf{v}_{n}\right)\right) \leq 2 \mathcal{E}\left(\pi^{*}\left(\mathbf{v}_{n}\right)\right),(15)
\end{array}
$$

where the last inequality comes from Theorem 1.

Note that the above result does not extend to general $k$ NNG dependency graphs $(k>1)$ for finite network size $n$. However, as the network size goes to infinity $(n \rightarrow \infty)$, we show in Section IV-B that a constant-factor approximation ratio is achieved by the DFMRF policy.

\section{ENERGY SCALING LAWS}

We now establish the scaling laws for optimal and suboptimal fusion policies. From the expression of average energy cost in (6), we see that the scaling laws rely on the law of large numbers (LLN) for stabilizing graph functionals. An overview of the LLN is provided in Appendix A.

We recall some notations and definitions used in this section. $X_{i} \stackrel{\text { i.i.d. }}{\sim} \tau$, where $\tau$ is supported on $Q_{1}$, the unit square centered at the origin $\mathbf{0}$. The node location-set is $\mathbf{V}_{n}:=\sqrt{\frac{n}{\lambda}}\left(X_{i}\right)_{i=1}^{n}$ and the limit is obtained by letting $n \rightarrow \infty$ with fixed $\lambda>0$. 


\section{A. Energy scaling for optimal fusion: independent case}

We first provide the scaling result for the case when the measurements are independent under either hypothesis. From Theorem 1, the optimal fusion policy minimizing the total energy consumption in (9) is given by aggregation along the directed minimum spanning tree. Hence, the energy scaling is obtained by the asymptotic analysis of the MST.

For the random node-location set $\mathbf{V}_{n}$, the average energy consumption of the optimal fusion policy for independent measurements is

$$
\overline{\mathcal{E}}\left(\pi^{*}\left(\mathbf{V}_{n}\right)\right)=\overline{\mathcal{E}}\left(\operatorname{MST}\left(\mathbf{V}_{n}\right)\right)=\frac{1}{n} \sum_{e \in \operatorname{MST}\left(\mathbf{V}_{n}\right)}|e|^{\nu}
$$

Let $\zeta(\nu ; \mathrm{MST})$ be the constant arising in the asymptotic analysis of the MST edge lengths, given by

$$
\zeta(\nu ; \operatorname{MST}):=\mathbb{E}\left[\sum_{e \in E\left(\mathbf{0} ; \operatorname{MST}\left(\mathcal{P}_{1} \cup\{\mathbf{0}\}\right)\right)} \frac{1}{2}|e|^{\nu}\right],
$$

where $\mathcal{P}_{a}$ is the homogeneous Poisson process of intensity $a>0$, and $E\left(\mathbf{0} ; \operatorname{MST}\left(\mathcal{P}_{1} \cup\{\mathbf{0}\}\right)\right)$ denotes the set of edges incident to the origin in $\operatorname{MST}\left(\mathcal{P}_{1} \cup\{\mathbf{0}\}\right)$. Hence, the above constant is half the expectation of the power-weighted edges incident to the origin in the minimum spanning tree over a homogeneous unit intensity Poisson process, and is discussed in Appendix A in (42). Although $\zeta(\nu ; \mathrm{MST})$ is not available in closed form, we evaluate it through simulations in Section V.

We now provide the scaling result for the optimal fusion policy when the measurements are independent based on the LLN for the MST obtained in [11, Thm 2.3(ii)].

Theorem 3 (Scaling for independent data [11]): When the sensor measurements are independent under each hypothesis, the limit of the average energy consumption of the optimal fusion policy in (16) is given by

$$
\lim _{n \rightarrow \infty} \overline{\mathcal{E}}\left(\pi^{*}\left(\mathbf{V}_{n}\right)\right) \stackrel{L^{2}}{=} \lambda^{-\frac{\nu}{2}} \zeta(\nu ; \mathrm{MST}) \int_{Q_{1}} \tau(x)^{1-\frac{\nu}{2}} d x
$$

Hence, asymptotically the average energy consumption of optimal fusion is a constant (independent of $n$ ) in the meansquare sense for independent measurements. In contrast, forwarding all the raw data to the fusion center according to the shortest-path (SP) policy has an unbounded average energy growing in the order of $\sqrt{n}$. Hence, significant energy savings are achieved through data fusion.

The scaling constant for average energy in (18) brings out the influence of several factors on energy consumption. It is inversely proportional to the node density $\lambda$. This is intuitive since placing the nodes with a higher density (i.e., in a smaller area) decreases the average inter-node distances and hence, also the energy consumption.

The node-placement pdf $\tau$ influences the asymptotic energy consumption through the term

$$
\int_{Q_{1}} \tau(x)^{1-\frac{\nu}{2}} d x
$$

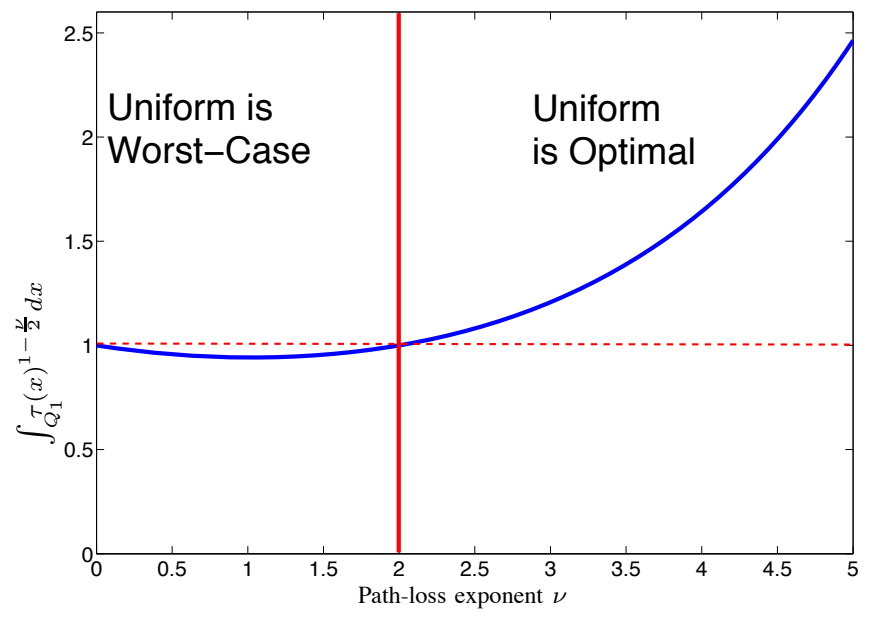

Fig. 3. Ratio of energy consumption under node placement distribution $\tau$ and uniform distribution as a function of path-loss exponent $\nu$. See (19) and (20).

When the placement is uniform $(\tau \equiv 1)$, the above term evaluates to unity. Hence, the scaling constant in (18) for uniform placement simplifies to

$$
\lambda^{-\frac{\nu}{2}} \zeta(\nu ; \mathrm{MST}) \text {. }
$$

The next theorem shows that the energy under uniform node placement $(\tau \equiv 1)$ optimizes the scaling limit in (18) when the path-loss exponent $\nu>2$. Also, see Fig.3.

Theorem 4 (Minimum energy placement: independent case): For any pdf $\tau$ supported on the unit square $Q_{1}$, we have

$$
\begin{aligned}
& \int_{Q_{1}} \tau(x)^{1-\frac{\nu}{2}} d x \geq 1, \quad \forall \nu>2, \\
& \int_{Q_{1}} \tau(x)^{1-\frac{\nu}{2}} d x \leq 1, \quad \forall \nu \in[0,2) .
\end{aligned}
$$

Proof: We have the Hölder inequality

$$
\left\|f_{1} f_{2}\right\|_{1} \leq\left\|f_{1}\right\|_{p}\left\|f_{2}\right\|_{q}, \quad \forall p>1, q=\frac{p}{p-1},
$$

where for any positive function $f$,

$$
\|f\|_{p}:=\left(\int_{Q_{1}} f(x)^{p} d x\right)^{\frac{1}{p}} .
$$

When $\nu>2$, in (21), substitute $f_{1}(x)$ with $\tau(x)^{\frac{1}{p}}, f_{2}(x)$ with $\tau(x)^{-\frac{1}{p}}$, and $p$ with $\frac{\nu}{\nu-2} \geq 1$ which ensures that $p>1$, to obtain (19).

For $\nu \in[0,2)$, in (21), substitute $f_{1}(x)$ with $\tau(x)^{\frac{1}{p}}, f_{2}(x)$ with $1, p=\frac{2}{2-\nu}>1$ to obtain (20).

The above result implies that, in the context of i.i.d. node placements, it is asymptotically energy-optimal to place the nodes uniformly when the path-loss exponent $\nu>2$, which is the case for wireless transmissions. The intuitive reason is as follows: without loss of generality, consider a clustered distribution in the unit square, where nodes are more likely to be placed near the origin. The MST over such a point set has many short edges, but a few very long edges, since a few nodes are placed near the boundary with finite probability. 
On the other hand, for uniform point sets, the edges of the MST are more likely to be all of similar lengths. Since for energy consumption, we have power-weighted edge-lengths with path-loss exponent $\nu>2$, long edges are penalized harshly, leading to higher energy consumption for clustered placement when compared with uniform node placement.

\section{B. Energy scaling for optimal fusion: MRF case}

We now evaluate the scaling laws for energy consumption of the DFMRF policy for a general Markov random field dependency among the sensor measurements. The DFMRF aggregation policy involves the cliques of the dependency graph which arise from correlation between the sensor measurements. Recall that the total energy consumption of DFMRF in (12) for random sensor locations $\mathbf{V}_{n}$ is given by

$$
\begin{aligned}
\mathcal{E}\left(\operatorname{DFMRF}\left(\mathbf{V}_{n}\right)\right)= & \sum_{c \in \mathcal{C}\left(\mathbf{V}_{n}\right)} \sum_{i \subset c} \mathcal{E}^{\mathrm{SP}}\left(i, \operatorname{Proc}(c) ; \mathcal{N}_{g}\right) \\
& +\mathcal{E}\left(\operatorname{MST}\left(\mathbf{V}_{n}\right)\right)
\end{aligned}
$$

where $\mathcal{E}^{\mathrm{SP}}\left(i, j ; \mathcal{N}_{g}\right)$ denotes the energy consumption for the shortest path between $i$ and $j$ using the links in the network graph $\mathcal{N}_{g}\left(\mathbf{V}_{n}\right)$ (set of feasible links for direct transmission).

We now additionally assume that the network graph $\mathcal{N}_{g}\left(\mathbf{V}_{n}\right)$ is a local $u$-energy spanner. In the literature [48], a graph $\mathcal{N}_{g}\left(\mathbf{V}_{n}\right)$ is called a $u$-energy spanner, for some constant $u>0$ called its energy stretch factor, when it satisfies

$$
\max _{i, j \in \mathbf{V}_{n}} \frac{\mathcal{E}^{\mathrm{SP}}\left(i, j ; \mathcal{N}_{g}\right)}{\mathcal{E}^{\mathrm{SP}}\left(i, j ; C_{g}\right)} \leq u,
$$

where $C_{g}\left(\mathbf{V}_{n}\right)$ denotes the complete graph on $\mathbf{V}_{n}$. In other words, the energy consumption between any two nodes is no worse than $u$-times the best possible value, i.e., over the shortest path using links in the complete graph. Intuitively, the $u$-spanning property ensures that the network graph possesses sufficient set of communication links to ensure that the energy consumed in the forwarding stage is bounded. Examples of energy $u$-spanners include the Gabriel graph ${ }^{12}$ (with stretch factor $u=1$ when the path-loss exponent $\nu \geq 2$ ), the Yao graph, and its variations [48]. In this paper, we only require a weaker version of the above property that asymptotically there is at most $u$-energy stretch between the neighbors in the dependency graph

$$
\limsup _{n \rightarrow \infty} \max _{(i, j) \in \mathcal{G}\left(\mathbf{V}_{n}\right)} \frac{\mathcal{E}^{\mathrm{SP}}\left(i, j ; \mathcal{N}_{g}\left(\mathbf{V}_{n}\right)\right)}{\mathcal{E}^{\mathrm{SP}}\left(i, j ; C_{g}\left(\mathbf{V}_{n}\right)\right)} \leq u .
$$

From (24), we have

$$
\begin{aligned}
\mathcal{E}\left(\mathrm{FG}\left(\mathbf{V}_{n}\right)\right) & \leq u \sum_{c \in \mathcal{C}\left(\mathbf{V}_{n}\right)} \sum_{i \subset c} \mathcal{E}^{\mathrm{sP}}\left(i, \operatorname{Proc}(c) ; C_{g}\right) \\
& \leq u \sum_{c \in \mathcal{C}\left(\mathbf{V}_{n}\right)} \sum_{i \subset c}|i, \operatorname{Proc}(c)|^{\nu}
\end{aligned}
$$

${ }^{12}$ The longest edge in Gabriel graph is $O(\sqrt{\log n})$, the same order as that of the MST [49]. Hence, the maximum power required at a node to ensure $u$-energy spanning property is of the same order as that needed for critical connectivity. where we use the property that the multihop shortest-path route from each node $i$ to $\operatorname{Proc}(c)$ consumes no more energy than the direct one-hop transmission.

In the DFMRF policy, recall that the processors are members of the respective cliques, i.e., $\operatorname{Proc}(c) \subset c$, for each clique $c$ in the dependency graph. Hence, in (25), only the edges of the processors of all the cliques are included in the summation. This is upper bounded by the sum of all the power-weighted edges of the dependency graph $\mathcal{G}\left(\mathbf{V}_{n}\right)$. Hence, we have

$$
\mathcal{E}\left(\mathrm{FG}\left(\mathbf{V}_{n}\right)\right) \leq u \sum_{e \in \mathcal{G}\left(\mathbf{V}_{n}\right)}|e|^{\nu}
$$

From (22), for the total energy consumption of the DFMRF policy, we have the upper bound,

$$
\mathcal{E}\left(\operatorname{DFMRF}\left(\mathbf{V}_{n}\right)\right) \leq u \sum_{e \in \mathcal{G}\left(\mathbf{V}_{n}\right)}|e|^{\nu}+\mathcal{E}\left(\operatorname{MST}\left(\mathbf{V}_{n}\right)\right)
$$

The above bound allows us to draw upon the general methods of asymptotic analysis for graph functionals presented in [11], [50].

From (27), the DFMRF policy scales whenever the righthand side of (26) scales. By Theorem 3, the energy consumption for aggregation along the MST scales. Hence, we only need to establish the scaling behavior of the first term in (26).

We now prove scaling laws governing the energy consumption of DFMRF and we also establish its asymptotic approximation ratio with respect to the optimal fusion policy. This in turn also establishes the scaling behavior of the optimal policy.

Theorem 5 (Scaling of DFMRF Policy): When the dependency graph $\mathcal{G}$ of the sensor measurements is either the $k$ nearest neighbor or the disc graph, the average energy of DFMRF policy satisfies

$$
\begin{aligned}
\limsup _{\substack{n \rightarrow \infty \\
\text { a.s. }}} \overline{\mathcal{E}}\left(\operatorname{DFMRF}\left(\mathbf{V}_{n}\right)\right) \\
\stackrel{\limsup _{n \rightarrow \infty}}{=}\left(\frac{1}{n} \sum_{e \in \mathcal{G}\left(\mathbf{V}_{n}\right)} u|e|^{\nu}+\overline{\mathcal{E}}\left(\operatorname{MST}\left(\mathbf{V}_{n}\right)\right)\right) \\
\quad \frac{u}{2} \int_{Q_{1}} \mathbb{E}\left[\sum_{j:(\mathbf{0}, j) \in \mathcal{G}\left(\mathcal{P}_{\lambda \tau(x)} \cup\{\mathbf{0}\}\right)}|\mathbf{0}, j|^{\nu}\right] \tau(x) d x \\
\quad+\lambda^{-\frac{\nu}{2}} \zeta(\nu ; \operatorname{MST}) \int_{Q_{1}} \tau(x)^{1-\frac{\nu}{2}} d x .
\end{aligned}
$$

Proof: See Appendix B.

Hence, the above result establishes the scalability of the DFMRF policy. In the theorem below, we use this result to prove the scalability of the optimal fusion policy and establish asymptotic upper and lower bounds on its average energy.

Theorem 6 (Scaling of Optimal Policy): When the dependency graph $\mathcal{G}$ is either the $k$-nearest neighbor or the disc graph, the limit of the average energy consumption of the optimal policy $\pi^{*}$ in (9) satisfies the upper bound

$$
\limsup _{n \rightarrow \infty} \bar{\varepsilon}\left(\pi^{*}\left(\mathbf{V}_{n}\right)\right) \stackrel{\text { a.s. }}{\leq} \limsup _{n \rightarrow \infty} \bar{\varepsilon}\left(\operatorname{DFMRF}\left(\mathbf{V}_{n}\right)\right)
$$


where the right-hand side satisfies the upper bound in (28). Also, $\pi^{*}$ satisfies the lower bound given by the MST

$$
\begin{aligned}
& \liminf _{n \rightarrow \infty} \overline{\mathcal{E}}\left(\operatorname{DFMRF}\left(\mathbf{V}_{n}\right)\right) \stackrel{\text { a.s. }}{\geq} \liminf _{n \rightarrow \infty} \overline{\mathcal{E}}\left(\pi^{*}\left(\mathbf{V}_{n}\right)\right) \\
& \stackrel{\text { a.s. }}{\geq} \lim _{n \rightarrow \infty} \overline{\mathcal{E}}\left(\operatorname{MST}\left(\mathbf{V}_{n}\right)\right) \stackrel{L^{2}}{=} \lambda^{-\frac{\nu}{2}} \zeta(\nu ; \operatorname{MST}) \int_{Q_{1}} \tau(x)^{1-\frac{\nu}{2}} d x .
\end{aligned}
$$

Proof: From (10), the DFMRF and the optimal policy satisfy the lower bound given by the MST.

Hence, the limiting average energy consumption for both the DFMRF policy and the optimal policy is strictly finite, and is bounded by (28) and (30). These bounds also establish that the approximation ratio of the DFMRF policy is asymptotically bounded by a constant, as stated below. Define the constant $\rho:=\rho(u, \lambda, \tau, \nu)$, given by

$$
\rho:=1+\frac{u \int_{Q_{1}} \frac{1}{2} \mathbb{E}\left[\sum_{j:(\mathbf{0}, j) \in \mathcal{G}\left(\mathcal{P}_{\lambda \tau(x)} \cup\{\mathbf{0}\}\right)}|\mathbf{0}, j|^{\nu}\right] \tau(x) d x}{\lambda^{-\frac{\nu}{2}} \zeta(\nu ; \operatorname{MST}) \int_{Q_{1}} \tau(x)^{1-\frac{\nu}{2}} d x} .
$$

Lemma 1 (Approximation Ratio for DFMRF): The approximation ratio of DFMRF is given by

$$
\begin{aligned}
& \limsup _{n \rightarrow \infty} \frac{\mathcal{E}\left(\operatorname{DFMRF}\left(\mathbf{V}_{n}\right)\right)}{\mathcal{E}\left(\pi^{*}\left(\mathbf{V}_{n}\right)\right)} \\
& \stackrel{\text { a.s. }}{\leq} \limsup _{n \rightarrow \infty} \frac{\mathcal{E}\left(\operatorname{DFMRF}\left(\mathbf{V}_{n}\right)\right)}{\mathcal{E}\left(\operatorname{MST}\left(\mathbf{V}_{n}\right)\right)} \stackrel{L^{2}}{=} \rho,
\end{aligned}
$$

where $\rho$ is given by (31).

Proof: Combine Theorem 5 and Theorem 6.

We further simplify the above results for the $k$-nearest neighbor dependency graph in the corollary below by exploiting its scale invariance. The results are expected to hold for other scale-invariant Euclidean stabilizing graphs as well. The edges of a scale-invariant graph are invariant under a change of scale, or put differently, $\mathcal{G}$ is scale invariant if scalar multiplication by any positive constant $\alpha$ from $\mathcal{G}\left(\mathbf{V}_{n}\right)$ to $\mathcal{G}\left(\alpha \mathbf{V}_{n}\right)$ induces a graph isomorphism for all node sets $\mathbf{V}_{n}$.

Along the lines of (17), let $\zeta(\nu ; k$-NNG) be the constant arising in the asymptotic analysis of the $k$-NNG edge lengths, that is

$$
\zeta(\nu ; k-\mathrm{NNG}):=\mathbb{E}\left[\sum_{j:(\mathbf{0}, j) \in k-\mathrm{NNG}\left(\mathcal{P}_{1} \cup\{\mathbf{0}\}\right)} \frac{1}{2}|\mathbf{0}, j|^{\nu}\right] .
$$

Corollary 1 ( $k-N N G$ Dependency Graph): We obtain a simplification of Theorem 5 and 6 for average energy consumption, namely

$$
\begin{aligned}
& \limsup _{n \rightarrow \infty} \overline{\mathcal{E}}\left(\pi^{*}\left(\mathbf{V}_{n}\right)\right) \stackrel{a . s .}{\leq} \limsup _{n \rightarrow \infty} \overline{\mathcal{E}}\left(\operatorname{DFMRF}\left(\mathbf{V}_{n}\right)\right) \\
& \stackrel{a . s .}{\leq} \limsup _{n \rightarrow \infty}\left(\frac{1}{n} \sum_{e \in \mathcal{G}\left(\mathbf{V}_{n}\right)} u|e|^{\nu}+\overline{\mathcal{E}}\left(\operatorname{MST}\left(\mathbf{V}_{n}\right)\right)\right) \\
& \stackrel{L^{2}}{=} \lambda^{-\frac{\nu}{2}}[u \zeta(\nu ; k-\mathrm{NNG})+\zeta(\nu ; \mathrm{MST})] \int_{Q_{1}} \tau(x)^{1-\frac{\nu}{2}} d x .
\end{aligned}
$$

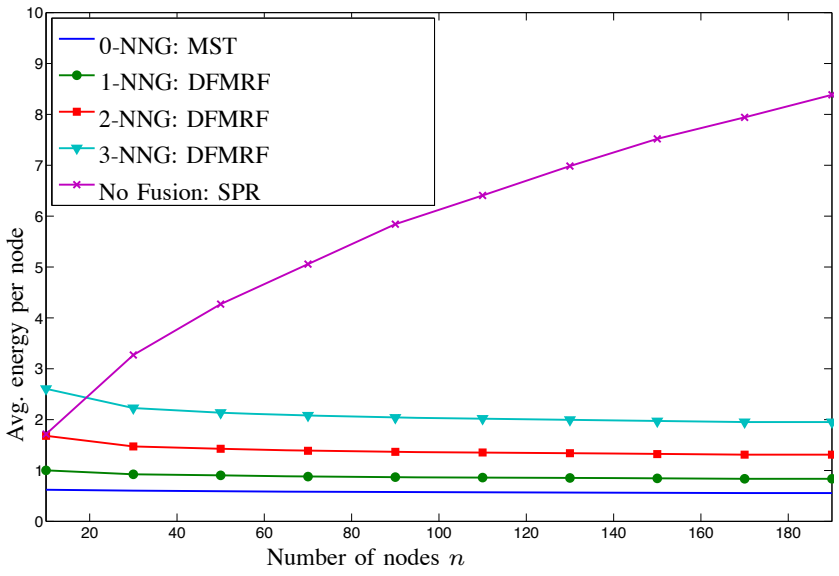

(a) Avg. energy vs. no. of nodes, $\nu=2$.

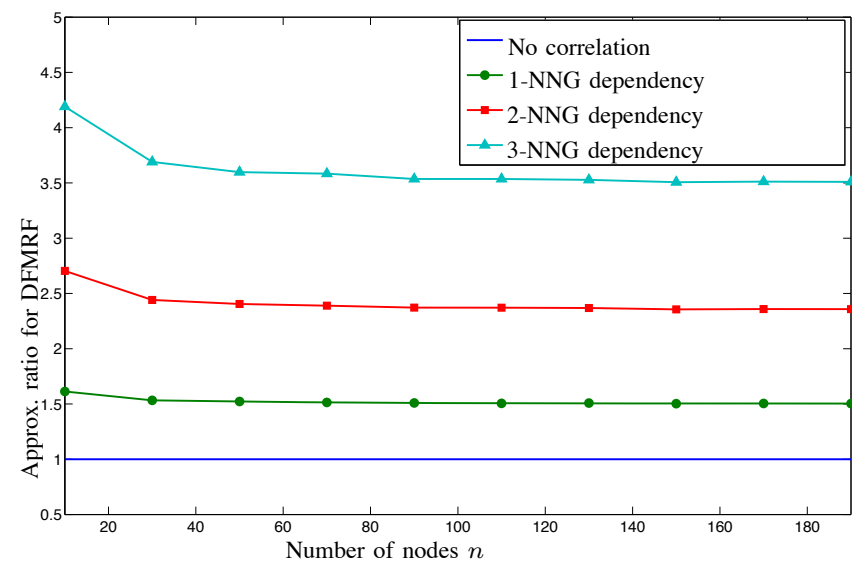

(b) Approx. ratio vs. no. of nodes, $\nu=2$.

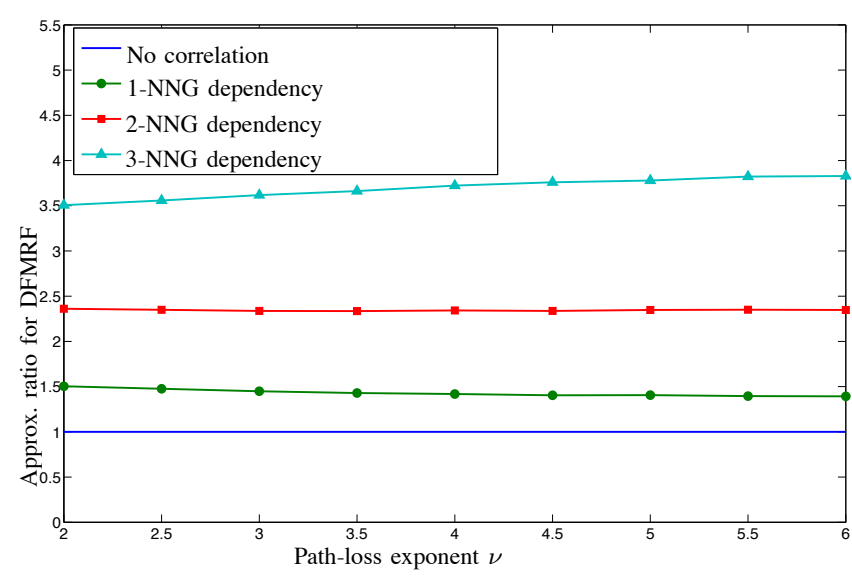

(c) Approx. ratio vs. path-loss, $n=190$.

Fig. 4. Average energy consumption for DFMRF policy and shortest-path routing for uniform node distribution and $k$-NNG dependency over 500 runs. Node density $\lambda=1$. See Corollary 1 .

The approximation ratio of DFMRF satisfies

$$
\begin{aligned}
& \limsup _{n \rightarrow \infty} \frac{\mathcal{E}\left(\operatorname{DFMRF}\left(\mathbf{V}_{n}\right)\right)}{\mathcal{E}\left(\pi^{*}\left(\mathbf{V}_{n}\right)\right)} \stackrel{\text { a.s. }}{\leq} \limsup _{n \rightarrow \infty} \frac{\mathcal{E}\left(\operatorname{DFMRF}\left(\mathbf{V}_{n}\right)\right)}{\mathcal{E}\left(\operatorname{MST}\left(\mathbf{V}_{n}\right)\right)} \\
& \stackrel{L^{2}}{=}\left(1+u \frac{\zeta(\nu ; k \text {-NNG })}{\zeta(\nu ; \mathrm{MST})}\right) .
\end{aligned}
$$

Proof: This follows from [11, Thm 2.2]. 


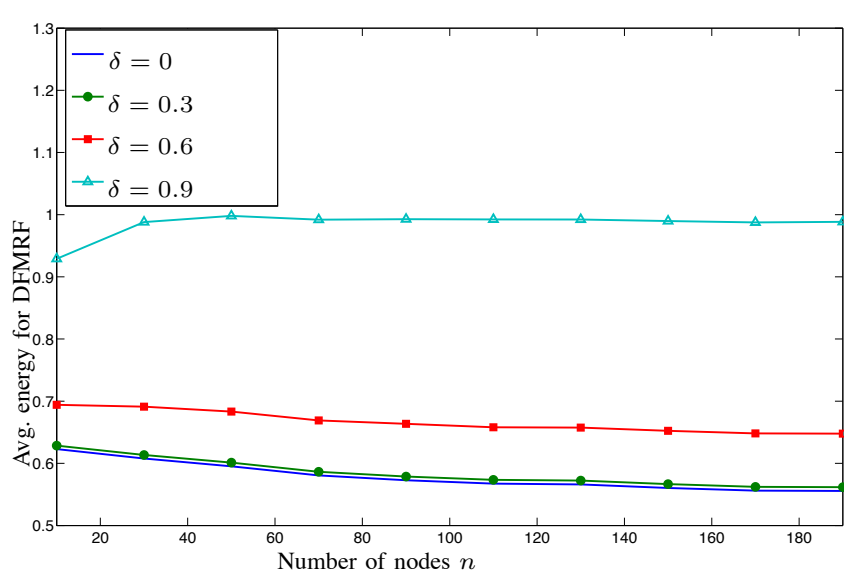

(a) Disk graph, $\nu=2$, uniform $(\tau \equiv 1)$.

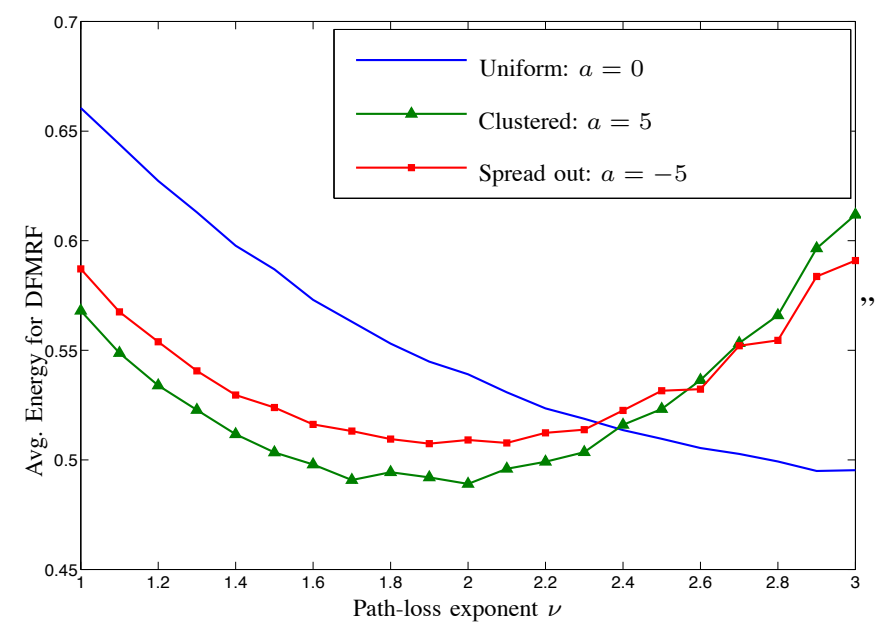

(b) Avg. energy vs. path loss, $\delta=0, n=190$.

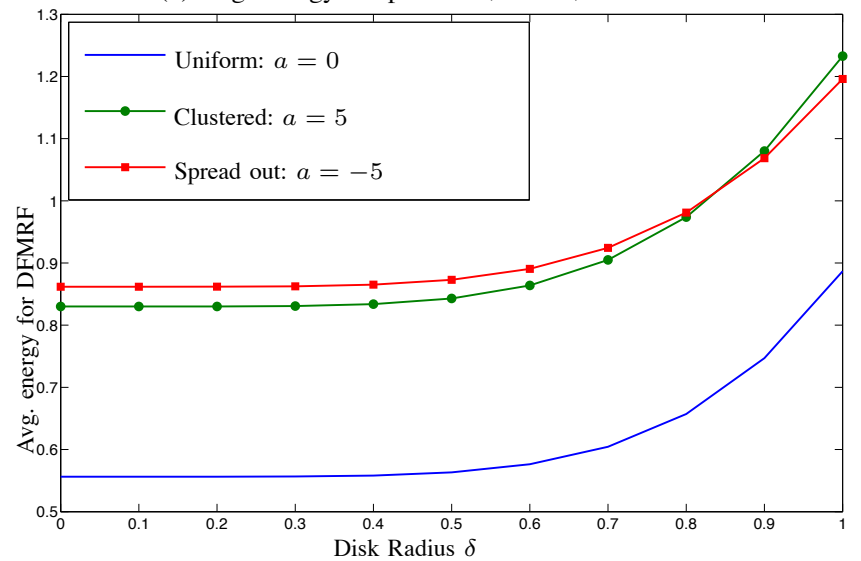

(c) Avg energy vs. disk radius, $\nu=4$.

Fig. 5. Average energy consumption for DFMRF policy over 500 runs for node-placement pdfs shown in Fig.6 under disc-dependency graph with radius $\delta$. Node density $\lambda=1$. See Theorem 5 .

Hence, the expressions for the energy scaling bounds and the approximation ratio are further simplified when the dependency graph is the $k$-nearest neighbor graph. A special case of this scaling result for the 1-nearest-neighbor dependency under uniform node placement was proven in [38, Thm 2].

It is interesting to note that the approximation factor for the $k$-NNG dependency graph in (35) is independent of the node placement pdf $\tau$ and node density $\lambda$. Hence, DFMRF has the same efficiency relative to the optimal policy under different node placements. The results of Theorem 4 on the optimality of the uniform node placement are also applicable here, but for the lower and upper bounds on energy consumption. We formally state it below.

Theorem 7 (Minimum energy bounds for $k-N N G$ ):

Uniform node placement $(\tau \equiv 1)$ minimizes the asymptotic lower and upper bounds on average energy consumption in (30) and (34) for the optimal policy under the $k$-NNG dependency graph over all i.i.d. node placement pdfs $\tau$.

Proof: From Theorem 4 and (34).

We also prove the optimality of uniform node-placement distribution under the disc-dependency graph, but over a limited set of node placement pdfs $\tau$.

Theorem 8 (Minimum energy bounds for disc graph): Uniform node placement $(\tau \equiv 1)$ minimizes the asymptotic lower and upper bounds on the average energy consumption in (30) and (34) for the optimal fusion policy under the disc dependency graph over all i.i.d. node-placement pdfs $\tau$ satisfying the lower bound

$$
\tau(x)>\frac{1}{\lambda}, \quad \forall x \in Q_{1},
$$

where $\lambda>1$ is the (fixed) node placement density.

Proof: We use the fact that for the disc graph $\mathcal{G}$ with a fixed radius, more edges are added as we scale down the area. Hence, for Poisson processes with intensities $\lambda_{1}>\lambda_{2}>0$,

$$
\mathbb{E}\left[\sum_{j:(\mathbf{0}, j) \in \mathcal{G}\left(\mathcal{P}_{\lambda_{1}} \cup\{\mathbf{0}\}\right)}|\mathbf{0}, j|^{\nu}\right] \geq \mathbb{E}\left[\sum_{j:(\mathbf{0}, j) \in \mathcal{G}\left(\mathcal{P}_{\lambda_{2}} \cup\{\mathbf{0}\}\right)}|\mathbf{0}, j|^{\nu}\right]\left[\frac{\lambda_{2}}{\lambda_{1}}\right]^{\frac{\nu}{2}},
$$

where the right-hand side is obtained by merely rescaling the edges present under the Poisson process at intensity $\lambda_{2}$. Since, new edges are added under the Poisson process at $\lambda_{1}$, the above expression is an inequality, unlike the case of $k$-NNG where the edge set is invariant under scaling. Substituting $\lambda_{1}$ with $\lambda \tau(x)$, and $\lambda_{2}$ by 1 under the condition that $\lambda \tau(x)>1$, $\forall x \in Q_{1}$, we have

$$
\begin{aligned}
& \left.\int_{Q_{1}} \underset{j:(\mathbf{0}, j) \in \mathcal{G}\left(\mathcal{P}_{\lambda \tau(x)} \cup\{\mathbf{0}\}\right)}{\mathbb{E}}\left|\sum\right| \mathbf{0},\left.j\right|^{\nu}\right] \tau(x) d x \\
\geq & \lambda^{-\frac{\nu}{2}} \mathbb{E}\left[\sum_{j:(\mathbf{0}, j) \in \mathcal{G}\left(\mathcal{P}_{1} \cup\{\mathbf{0}\}\right)}|\mathbf{0}, j|^{\nu}\right] \int_{Q_{1}} \tau(x)^{1-\frac{\nu}{2}} d x, \\
\geq & \lambda^{-\frac{\nu}{2}} \mathbb{E}\left[\sum_{j:(\mathbf{0}, j) \in \mathcal{G}\left(\mathcal{P}_{1} \cup\{\mathbf{0}\}\right)}|\mathbf{0}, j|^{\nu}\right], \quad \nu>2 .
\end{aligned}
$$

Hence, uniform node placement is optimal in terms of the energy scaling bounds under the disc dependency graph if we restrict to pdfs $\tau$ satisfying (36).

We have so far established the finite scaling of the average energy when the dependency graph describing the correlations among the sensor observations is either the $k$-NNG or the disc graph with finite radius. However, we cannot expect finite energy scaling under any general dependency graph. For instance, when the dependency graph is the complete graph, the log-likelihood ratio in (7) is a function of only one clique containing all the nodes. In this case, the optimal 
policy in (9) consists of a unique processor chosen optimally, to which all the other nodes forward their raw data along shortest paths, and the processor then forwards the value of the computed log-likelihood ratio to the fusion center. Hence, for the complete dependency graph, the optimal fusion policy reduces to a version of the shortest-path (SP) routing, where the average energy consumption grows as $\sqrt{n}$ and does not scale with $n$.

\section{NUMERICAL ILlUSTRATIONS}

As described in Section II-A, $n$ nodes are placed in area $\frac{n}{\lambda}$ and one of them is randomly chosen as the fusion center. We conduct 500 independent simulation runs and average the results. We fix node density $\lambda=1$. We plot results for two cases of dependency graph, viz., the $k$-nearest neighbor graph and the disc graph with a fixed radius $\delta$.

In Fig.4, we plot the simulation results for the $k$-nearest neighbor dependency graph and uniform node placement. Recall in Corollary 1, we established that the average energy consumption of the DFMRF policy in (34) is finite and bounded for asymptotic networks under $k$-NNG dependency. On the other hand, we predicted in Section I-A that the average energy under no aggregation (SP policy) increases without bound with the network size. The results in Fig.4a agree with our theory and we note that the convergence to asymptotic values is quick, and occurs in networks with as little as 30 nodes. We also see that the energy for DFMRF policy increases with the number of neighbors $k$ in the dependency graph since the graph has more edges leading to computation of a more complex likelihood ratio by the DFMRF policy.

We plot the approximation ratio of the DFMRF policy for $k$-NNG in (35) against the number of nodes in Fig.4b and against the path-loss exponent $\nu$ in Fig.4c. As established by Corollary 1 , the approximation ratio is a constant for large networks, and we find a quick convergence to this value in Fig.4b as we increase the network size. In Fig.4c, we also find that the approximation ratio is fairly insensitive with respect to the path-loss exponent $\nu$.

In Fig.5a, we plot the average energy consumption of DFMRF in (28) under uniform node placement and the disc dependency graph with radius $\delta$. The average energy is bounded, as established by Theorem 5 . As in the $k$-NNG case, on increasing the network size, there is a quick convergence to the asymptotic values. Moreover, as expected, energy consumption increases with the radius $\delta$ of the disc graph since there are more edges. Note that the energy consumption at $\delta=0$ and $\delta=0.3$ are nearly the same, since at $\delta=0.3$, the disc graph is still very sparse, and hence, the energy consumed in the forwarding stage of the likelihood-ratio computation is small.

We now study the effect of i.i.d. node-placement $\operatorname{pdf} \tau$ on the energy consumption of both DFMRF policy and shortestpath policy with no data aggregation. In Fig.5b, Fig.5c and Fig.7, we consider a family of truncated-exponential pdfs $\tau_{a}$ given by

$$
\tau_{a}(x)=\xi_{a}(x(1)) \xi_{a}(x(2)), \quad x \in \mathbb{R}^{2},
$$

where, for some $a \neq 0, \xi_{a}$ is given by the truncated exponential

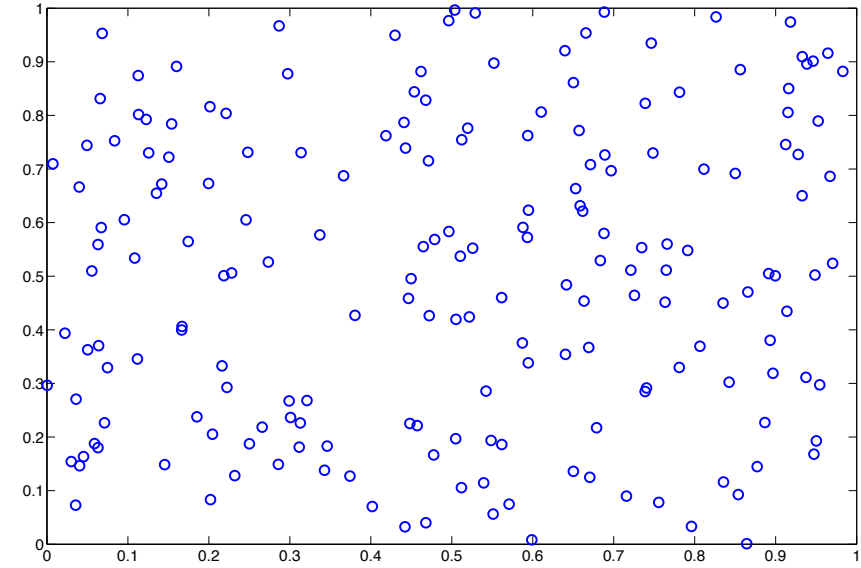

(a) Uniform $a \rightarrow 0$.

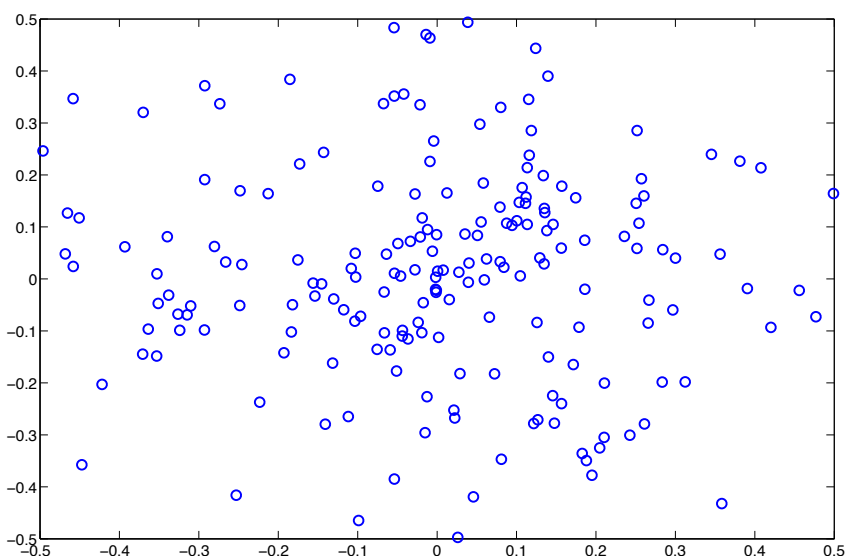

(b) Clustered $a=5$.

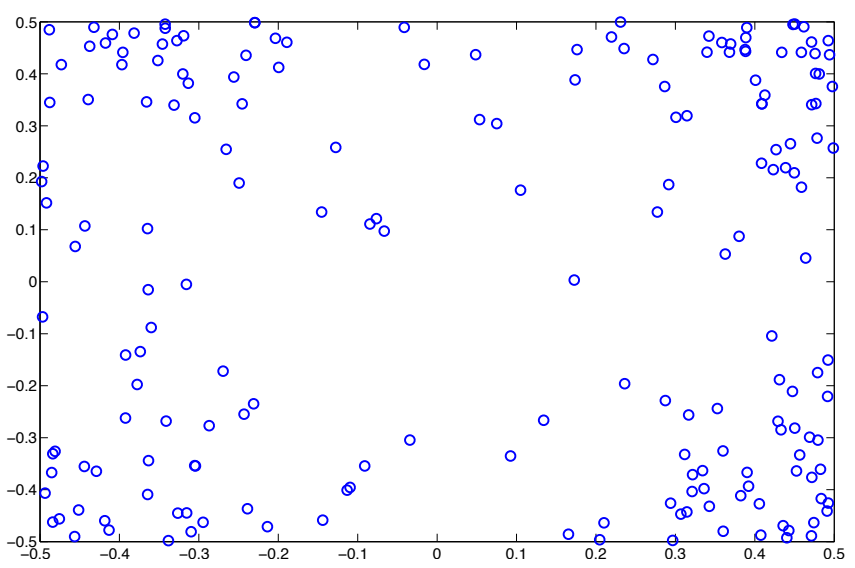

(c) Spread-out $a=-5$.

Fig. 6. Sample realization of $n=190$ points on unit square. See (37), (38).

$$
\xi_{a}(z):= \begin{cases}\frac{a e^{-a|z|}}{2\left(1-e^{-\frac{a}{2}}\right)}, & \text { if } z \in\left[-\frac{1}{2}, \frac{1}{2}\right], \\ 0, & \text { o.w. }\end{cases}
$$

Note that as $a \rightarrow 0$, we obtain the uniform distribution in the limit $\left(\tau_{0} \equiv 1\right)$. A positive $a$ corresponds to clustering of the points with respect to the origin and viceversa. In Fig.6, a sample realization is shown for the cases $a= \pm 5$ and $a \rightarrow 0$.

Intuitively, for shortest-path (SP) policy where there is no data aggregation, the influence of node placement on the 


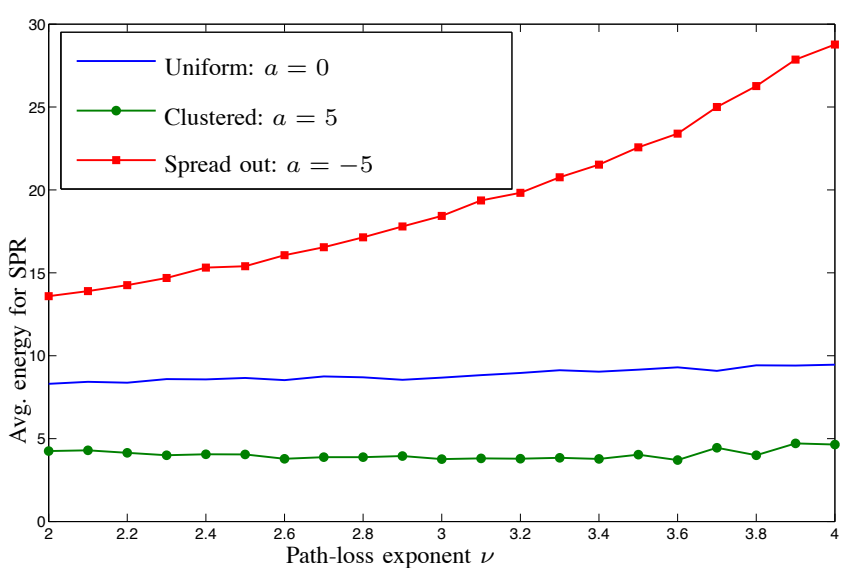

Fig. 7. Average energy for shortest-path routing policy over 500 runs for node-placement pdfs shown in Fig.6 and number of nodes $n=190$.

energy consumption is fairly straightforward. If we cluster the nodes close to one another, the average energy consumption decreases. On the other hand, spreading the nodes out towards the boundary increases the average energy. Indeed, we observe this behavior in Fig.7, for the placement pdf $\tau_{a}$ defined above in (37) and (38). However, as established in the previous sections, optimal node placement for the DFMRF policy does not follow this simple intuition.

In Theorem 4, we established that the uniform node placement $\left(\tau_{0} \equiv 1\right)$ minimizes the asymptotic average energy consumption of the optimal policy (which turns out to be the DFMRF policy), when the path-loss exponent $\nu \geq 2$. For $\nu \in[0,2]$, the uniform distribution has the worst-case value. This is verified in Fig.5b, where for $\nu \in[1,3]$, the uniform distribution initially has high energy consumption but decreases as we increase the path-loss exponent $\nu$. We see that at threshold of around $\nu=2.4$, the uniform distribution starts having lower energy than the non-uniform placements (clustered and spread-out), while according to Theorem 4, the threshold should be $\nu=2$. Moreover, Theorem 4 also establishes that the clustered and spread-out distributions $(a \pm 5)$ have the same energy consumption since the expressions $\int_{Q_{1}} \tau_{a}(x)^{1-\frac{\nu}{2}} d x$ for $a=5$ and $a=-5$ are equal for $\tau_{a}$ given by (37) and (38), and this approximately holds in Fig.5b.

We now study the energy consumption of the DFMRF policy in Fig.5c under the disc dependency graph and the node placements given in Fig.6. In Fig.5c, for path-loss exponent $\nu=4$, we find that the uniform node placement $\left(\tau_{0} \equiv 1\right)$ performs significantly better than the non-uniform placements for the entire range of the disc radius $\delta$. Intuitively, this is because at large path-loss exponent $\nu$, communication over long edges consumes a lot of energy and long edges occur with higher probability in non-uniform placements (both clustered and spread-out) compared to the uniform placement. Hence, uniform node placement is significantly energy-efficient under high path-loss exponent of communication.

\section{CONCLUSION}

We analyzed the scaling laws for energy consumption of data-fusion policies under the constraint of optimal statistical inference at the fusion center. Forwarding all the raw data without fusion has an unbounded average energy as we increase the network size, and hence, is not a feasible strategy in energy-constrained networks. We established finite average energy scaling for a fusion policy known as the Data Fusion for Markov Random Fields (DFMRF) for a class of spatial correlation model. We analyzed the influence of the correlation structure given by the dependency graph, the node placement distribution and the transmission environment (path-loss exponent) on the energy consumption.

There are many issues which are not handled in this paper. Our fusion policy DFMRF needs centralized network information for constructed, and we plan to investigate distributed policies when only local information is available at the nodes. Our model currently only incorporates i.i.d. node placements and we expect our results to extend to the correlated node placement according to a Gibbs point process through the results in [51]. We have not considered here the scaling behavior of the inference accuracy (error probability) with network size, and this is a topic of study in [39], [40]. We have not considered the time required for data fusion, and it is interesting to establish bounds in this case. Our current correlation model assumes a discrete Markov random field. A more natural but difficult approach is to consider Markov field over a continuous space [52] and then, sample it through node placements.

\section{Acknowledgment}

The authors thank A. Ephremides, T. He, D. Shah, the guest editor M. Haenggi and the anonymous reviewers for helpful comments.

\section{APPENDIX}

\section{A. Functionals on random points sets}

In [11], [41], [53], Penrose and Yukich introduce the concept of stabilizing functionals to establish weak laws of large numbers for functionals on graphs with random vertex sets. As in this paper, the vertex sets may be marked (sensor measurements constituting one example of marks), but for simplicity of exposition we work with unmarked vertices. We briefly describe the general weak law of large numbers after introducing the necessary definitions.

Graph functionals on a vertex set $\mathbf{V}$ are often represented as sums of spatially dependent terms

$$
\sum_{x \in \mathbf{V}} \xi(x, \mathbf{V}),
$$

where $\mathbf{V} \subset \mathbb{R}^{2}$ is locally finite (contains only finitely many points in any bounded region), and the measurable function $\xi$, defined on all pairs $(x, \mathbf{V})$, with $x \in \mathbf{V}$, represents the interaction of $x$ with other points in $\mathbf{V}$. We see that the functionals corresponding to energy consumption can be cast in this framework.

When $\mathbf{V}$ is random, the range of spatial dependence of $\xi$ at node $x \in \mathbf{V}$ is random, and the purpose of stabilization is to quantify this range in a way useful for asymptotic analysis. There are several similar notions of stabilization, but the essence is captured by the notion of stabilization of $\xi$ with respect to homogeneous Poisson points on $\mathbb{R}^{2}$, defined 
as follows. Recall that $\mathcal{P}_{a}$ is a homogeneous Poisson point process with intensity $a>0$.

We say that $\xi$ is translation invariant if $\xi(x, \mathbf{V})=\xi(x+$ $z, \mathbf{V}+z)$ for all $z \in \mathbb{R}^{2}$. Let $\mathbf{0}$ denote the origin of $\mathbb{R}^{2}$ and let $B_{r}(x)$ denote the Euclidean ball centered at $x$ with radius $r$. A translation-invariant $\xi$ is homogeneously stabilizing if for all intensities $a>0$ there exists almost surely a finite random variable $R:=R(a)$ such that

$$
\xi\left(\mathbf{0},\left(\mathcal{P}_{a} \cap B_{R}(\mathbf{0})\right) \cup \mathcal{A}\right)=\xi\left(\mathbf{0}, \mathcal{P}_{a} \cap B_{R}(\mathbf{0})\right)
$$

for all locally finite $\mathcal{A} \subset \mathbb{R}^{2} \backslash B_{R}(\mathbf{0})$. Thus $\xi$ stabilizes if the value of $\xi$ at $\mathbf{0}$ is unaffected by changes in point configurations outside $B_{R}(\mathbf{0})$.

$\xi$ satisfies the moment condition of order $p>0$ if

$$
\sup _{n \in \mathbb{N}} \mathbb{E}\left[\xi\left(n^{\frac{1}{2}} X_{1}, n^{\frac{1}{2}}\left\{X_{i}\right\}_{i=1}^{n}\right)^{p}\right]<\infty .
$$

We use the following weak laws of large numbers throughout. Recall that $X_{i}$ are i.i.d. with density $\tau$.

Theorem 9 (WLLN [11], [50]): Put $q=1$ or $q=2$. Let $\xi$ be a homogeneously stabilizing translation-invariant functional satisfying the moment condition (39) for some $p>q$. Then

$$
\begin{aligned}
& \lim _{n \rightarrow \infty} \frac{1}{n} \sum_{i=1}^{n} \xi\left(\sqrt{\frac{n}{\lambda}} X_{i}, \sqrt{\frac{n}{\lambda}}\left\{X_{j}\right\}_{j=1}^{n}\right) \\
= & \int_{Q_{1}} \mathbb{E}\left[\xi\left(\mathbf{0}, \mathcal{P}_{\lambda \tau(x)}\right)\right] \tau(x) d x \text { in } L^{q} .
\end{aligned}
$$

We interpret the right-hand side of the above equation as a weighted average of the values of $\xi$ on homogeneous Poisson point processes $\mathcal{P}_{\lambda \tau(x)}$. When $\xi$ satisfies scaling such as $\mathbb{E}\left[\xi\left(\mathbf{0}, \mathcal{P}_{a}\right)\right]=a^{-\alpha} \mathbb{E}\left[\xi\left(\mathbf{0}, \mathcal{P}_{1}\right)\right]$, then the limit on the righthand side of (40) simplifies to

$$
\lambda^{-\alpha} \mathbb{E}\left[\xi\left(\mathbf{0}, \mathcal{P}_{1}\right)\right] \int_{Q_{1}}(\tau(x))^{1-\alpha} d x \text { in } L^{q},
$$

a limit appearing regularly in problems in Euclidean combinatorial optimization. For uniform node placement $(\tau(x) \equiv 1)$, the expression in (40) reduces to $\mathbb{E}\left[\xi\left(\mathbf{0}, \mathcal{P}_{\lambda}\right)\right]$, and the LLN result for this instance is pictorially depicted in Fig.8.

For example, if $\xi(x, \mathbf{V})$ is one half the sum of the $\nu$ power weighted edges incident to $x$ in the MST (or any scaleinvariant stabilizing graph) on $\mathbf{V}$, i.e.,

$$
\xi(x, \mathbf{V}):=\frac{1}{2} \sum_{e \in E(x, \operatorname{MST}(\mathbf{V}))}|e|^{\nu},
$$

then substituting $\alpha$ with $\frac{\nu}{2}$ in (41),

$$
\begin{aligned}
& \lim _{n \rightarrow \infty} \frac{1}{n} \sum_{i=1}^{n} \xi\left(\sqrt{\frac{n}{\lambda}} X_{i}, \sqrt{\frac{n}{\lambda}}\left\{X_{i}\right\}_{i=1}^{n}\right) \\
= & \lambda^{-\frac{\nu}{2}} \mathbb{E}\left[\xi\left(\mathbf{0}, \mathcal{P}_{1}\right)\right] \int_{Q_{1}}(\tau(x))^{1-\frac{\nu}{2}} d x \\
= & \lambda^{-\frac{\nu}{2}} \zeta(\nu ; \operatorname{MST}) \int_{Q_{1}}(\tau(x))^{1-\frac{\nu}{2}} d x,
\end{aligned}
$$

where $\zeta(\nu ;$ MST $)$ is defined in (17).

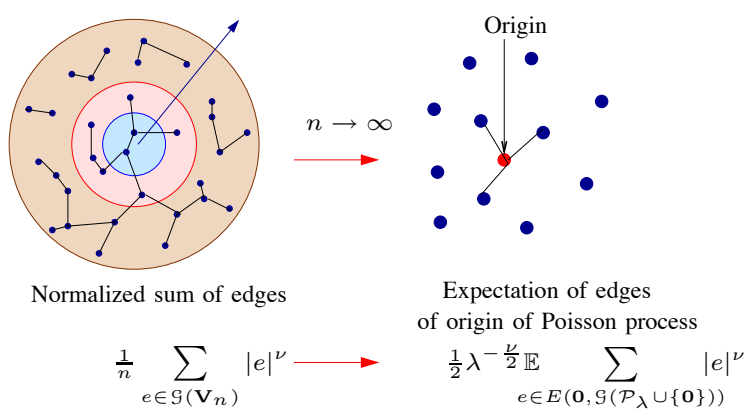

Fig. 8. LLN for sum graph edges on uniform point sets $(\tau \equiv 1)$.

\section{B. Proof of Theorem 5}

The energy consumption of DFMRF satisfies the inequality in (28). For the MST we have the result in Theorem 3. We now use stabilizing functionals to show that

$$
\frac{1}{n} \sum_{e \in \mathcal{G}\left(\mathbf{V}_{n}\right)}|e|^{\nu}
$$

converges in $L^{2}$ to a constant. For all locally finite vertex sets $\mathcal{X} \subset \mathbb{R}^{2}$ supporting some dependency graph $\mathcal{G}(\mathcal{X})$ and for all $x \in \mathcal{X}$, define the functional $\eta(x, \mathcal{X})$ by

$$
\eta(x, \mathcal{X}):=\sum_{y:(x, y) \in \mathcal{G}(\mathcal{X})}|x, y|^{\nu} .
$$

Notice that $\sum_{x \in \mathcal{X}} \eta(x, \mathcal{X})=2 \sum_{e \in \mathcal{G}(\mathcal{X})}|e|^{\nu}$.

From [11, Thm 2.4], the sum of power-weighted edges of the $k$-nearest neighbors graph is a stabilizing functional and satisfies the bounded-moments condition (39). Hence, the limit in (40) holds when the dependency graph is the $k$-NNG.

Finally, the sum of power-weighted edges of the continuum percolation graph is a stabilizing functional which satisfies the bounded-moments condition (39), thus implying that the limit in (40) holds.

Indeed, $\eta$ stabilizes with respect to $\mathcal{P}_{a}, a \in(0, \infty)$, since points distant from $x$ by more than the deterministic disc radius do not modify the value of $\eta\left(x, \mathcal{P}_{a}\right)$. Moreover, $\eta$ satisfies the bounded moments condition (39) since each $|x, y|$ is bounded by the deterministic disc radius and the number of nodes in $n^{\frac{1}{2}}\left\{X_{i}\right\}_{i=1}^{n}$ which are joined to $n^{\frac{1}{2}} X_{1}$ is a random variable with moments of all orders.

\section{REFERENCES}

[1] A. Anandkumar, J.E.Yukich, A. Swami, and L. Tong, "EnergyPerformance Scaling Laws for Statistical Inference in Large Random Networks," in Proc. ASA Joint Stat. Meet., Denver, USA, Aug. 2008.

[2] A. Anandkumar, J. Yukich, L. Tong, and A. Swami, "Scaling Laws for Statistical Inference in Random Networks," in Proc. Allerton Conf. on Communication, Control and Computing, Monticello, USA, Sept. 2008.

[3] A. Ephremides, "Energy Concerns in Wireless Networks," IEEE Wireless Commun., no. 4, pp. 48-59, August 2002.

[4] P.Billingsley, Probability and Measure. New York, NY: Wiley InterScience, 1995.

[5] W. Li and H. Dai, "Energy-Efficient Distributed Detection Via Multihop Transmission in Sensor Networks," IEEE Signal Processing Letters, vol. 15 , pp. 265-268, 2008.

[6] A. Anandkumar, M. Wang, L. Tong, and A. Swami, "Prize-Collecting Data Fusion for Cost-Performance Tradeoff in Distributed Inference," in Proc. IEEE INFOCOM, Rio De Janeiro, Brazil, April 2009.

[7] A. Anandkumar, L. Tong, A. Swami, and A. Ephremides, "Minimum Cost Data Aggregation with Localized Processing for Statistical Inference," in Proc. INFOCOM, Phoenix, USA, April 2008, pp. 780-788. 
[8] J. Steele, "Growth Rates of Euclidean Minimal Spanning Trees with Power Weighted Edges," The Annals of Probability, vol. 16, no. 4, pp. 1767-1787, 1988.

[9] J. Yukich, "Asymptotics for Weighted Minimal Spanning Trees on Random Points," Stochastic Processes and their Applications, vol. 85, no. 1 , pp. $123-138,2000$.

[10] D. Aldous and J. Steele, "The objective method: probabilistic combinatorial optimization and local weak convergence," Probability on Discrete Structures, vol. 110, pp. 1-72, 2004.

[11] M. Penrose and J. Yukich, "Weak Laws Of Large Numbers In Geometric Probability," Annals of Applied Probability, vol. 13, no. 1, pp. 277-303, 2003.

[12] A. Anandkumar, L. Tong, and A. Swami, "Energy Efficient Routing for Statistical Inference of Markov Random Fields," in Proc. CISS '07, Baltimore, USA, March 2007, pp. 643-648.

[13] P. Gupta and P. R. Kumar, "The Capacity of Wireless Networks," IEEE Tran. Inform. Theory, vol. 46, no. 2, pp. 388-404, March 2000.

[14] M. Franceschetti and R. Meester, Random Networks for Communication: From Statistical Physics to Information Systems. Cambridge University Press, 2008.

[15] Q. Zhao and L. Tong, "Energy Efficiency of Large-Scale Wireless Networks: Proactive vs. Reactive Networking," IEEE J. Select. Areas Commun. Special Issue on Advances in Military Wireless Communications, May 2005.

[16] X. Liu and M. Haenggi, "Toward Quasiregular Sensor Networks: Topology Control Algorithms for Improved Energy Efficiency," IEEE Trans. Parallel Distrib. Syst., pp. 975-986, 2006.

[17] X. Wu, G. Chen, and S. Das, "Avoiding Energy Holes in Wireless Sensor Networks with Nonuniform Node Distribution," IEEE Trans. Parallel Distrib. Syst., vol. 19, no. 5, pp. 710-720, May 2008.

[18] Q. Zhao, A. Swami, and L. Tong, "The Interplay Between Signal Processing and Networking in Sensor Networks," IEEE Signal Processing Mag., vol. 23, no. 4, pp. 84-93, 2006.

[19] A. Giridhar and P. Kumar, "Toward a Theory of In-network Computation in Wireless Sensor Networks," IEEE Commun. Mag., vol. 44, no. 4, pp. 98-107, 2006.

[20] R. Cristescu, B. Beferull-Lozano, M. Vetterli, and R. Wattenhofer, "Network Correlated Data Gathering with Explicit Communication: NPCompleteness and Algorithms," IEEE/ACM Trans. Networking, vol. 14, no. 1, pp. 41-54, 2006.

[21] P. von Rickenbach and R. Wattenhofer, "Gathering Correlated Data in Sensor Networks," in Joint workshop on Foundations of Mobile Computing, 2004, pp. 60-66.

[22] H. Gupta, V. Navda, S. Das, and V. Chowdhary, "Efficient gathering of correlated data in sensor networks," in Proc. ACM Intl. symposium on Mobile ad hoc networking and computing, 2005, pp. 402-413.

[23] S. Madden, M. Franklin, J. Hellerstein, and W. Hong, "TinyDB: an acquisitional query processing system for sensor networks," ACM Trans. Database Systems, vol. 30, no. 1, pp. 122-173, 2005.

[24] C. Intanagonwiwat, R. Govindan, and D. Esterin, "Directed Diffusion : A Scalable and Robust Paradigm for Sensor Networks," in Proc. 6th ACM/Mobicom Conference, Boston,MA, 2000, pp. pp 56-67.

[25] B. Krishnamachari, D. Estrin, and S. Wicker, "Modeling Data-centric Routing in Wireless Sensor Networks," in IEEE INFOCOM, New York, USA, 2002.

[26] A. Giridhar and P. Kumar, "Maximizing the functional lifetime of sensor networks," in Proc. of IPSN, 2005.

[27] - -, "Computing and Communicating Functions over Sensor Networks," IEEE JSAC, vol. 23, no. 4, pp. 755-764, 2005.

[28] S. Subramanian, P. Gupta, and S. Shakkottai, "Scaling Bounds for Function Computation over Large Networks," in IEEE ISIT, June 2007.

[29] O. Ayaso, D. Shah, and M. Dahleh, "Counting Bits for Distributed Function Computation," in Proc. ISIT, Toronto, Canada, July 2008, pp. 652-656.

[30] Y. Sung, S. Misra, L. Tong, and A. Ephremides, "Cooperative Routing for Signal Detection in Large Sensor Networks," IEEE J. Select. Areas Commun., vol. 25, no. 2, pp. 471-483, 2007.

[31] J. Chamberland and V. Veeravalli, "How Dense Should a Sensor Network Be for Detection With Correlated Observations?" IEEE Trans. Inform. Theory, vol. 52, no. 11, pp. 5099-5106, 2006.

[32] S. Misra and L. Tong, "Error Exponents for Bayesian Detection with Randomly Spaced Sensors," IEEE Trans. Signal Processing, vol. 56, no. 8, 2008.

[33] Y. Sung, X. Zhang, L. Tong, and H. Poor, "Sensor Configuration and Activation for Field Detection in Large Sensor Arrays," IEEE Trans. Signal Processing, vol. 56, no. 2, pp. 447-463, 2008.
[34] Y. Sung, H. Yu, and H. V. Poor, "Information, Energy and Density for Ad-hoc Sensor Networks over Correlated Random Fields: Largedeviation Analysis," in IEEE ISIT, July 2008, pp. 1592-1596.

[35] N. Katenka, E. Levina, and G. Michailidis, "Local Vote Decision Fusion for Target Detection in Wireless Sensor Networks," in Joint Research Conf. on Statistics in Quality Industry and Tech., Knoxville, USA, June 2006.

[36] L. Yu, L. Yuan, G. Qu, and A. Ephremides, "Energy-driven Detection Scheme with Guaranteed Accuracy," in Proc. IPSN, 2006, pp. 284-291.

[37] A. Anandkumar, A. Ephremides, A. Swami, and L. Tong, "Routing for Statistical Inference in Sensor Networks," in Handbook on Array Processing and Sensor Networks, S. Haykin and R. Liu, Eds. John Wiley \& Sons, 2009, ch. 23.

[38] A. Anandkumar, L. Tong, and A. Swami, "Optimal Node Density for Detection in Energy Constrained Random Networks," IEEE Trans. Signal Processing, vol. 56, no. 10, pp. 5232-5245, Oct. 2008.

[39] - - "Detection of Gauss-Markov Random Fields with Nearestneighbor Dependency," IEEE Trans. Inform. Theory, vol. 55, no. 2, pp. 816-827, Feb. 2009.

[40] A. Anandkumar, J. Yukich, L. Tong, and A. Willsky, "Detection Error Exponent for Spatially Dependent Samples in Random Networks," in Proc. of IEEE ISIT, Seoul, S. Korea, July 2009.

[41] M. Penrose and J. Yukich, "Limit Theory For Random Sequential Packing And Deposition," Annals of Applied probability, vol. 12, no. 1, pp. 272-301, 2002.

[42] M. Penrose, Random Geometric Graphs. Oxford University Press, 2003.

[43] P. Brémaud, Markov Chains: Gibbs fields, Monte Carlo simulation, and queues. Springer, 1999.

[44] P. Clifford, "Markov Random Fields in Statistics," Disorder in Physical Systems, pp. 19-32, 1990.

[45] M. Jerrum and A. Sinclair, "Polynomial Time Approximations for the Ising Model,” SIAM J. Computing, vol. 22, no. 5, pp. 1087-1116, 1993.

[46] H. V. Poor, An Introduction to Signal Detection and Estimation. New York: Springer-Verlag, 1994

[47] E. Dynkin, "Necessary and Sufficient Statistics for a Family of Probability Distributions," Tran. Math, Stat. and Prob., vol. 1, pp. 23-41, 1961.

[48] X. Li, "Algorithmic, Geometric and Graphs Issues in Wireless Networks," Wireless Comm. and Mobile Computing, vol. 3, no. 2, March 2003.

[49] P. Wan and C. Yi, "On the Longest Edge of Gabriel Graphs in Wireless Ad Hoc Networks," IEEE Trans. Parallel Distrib. Syst., pp. 111-125, 2007.

[50] M. Penrose, "Laws Of Large Numbers In Stochastic Geometry With Statistical Applications," Bernoulli, vol. 13, no. 4, pp. 1124-1150, 2007.

[51] T. Schreiber and J. Yukich, "Stabilization and Limit Theorems for Geometric Functionals of Gibbs Point Processes," Arxiv preprint arXiv:0802.0647, 2008.

[52] H. Künsch, "Gaussian Markov Random Fields," J. Fac. Sci. Univ. of Tokyo, no. 26, pp. 53-73, 1979.

[53] M. Penrose and J. Yukich, "Central Limit Theorems For Some Graphs In Computational Geometry," Annals of Applied Probability, vol. 11, no. 4 , pp. 1005-1041, 2001. 


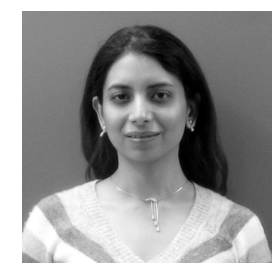

Animashree Anandkumar (S '02) received her B.Tech in Electrical Engineering from the Indian Institute of Technology Madras in 2004 with a minor in Theoretical Computer Science. She is a $\mathrm{PhD}$ student in Electrical Engineering at Cornell University with a minor in Applied Mathematics. Since Fall 2008, she is visiting the Stochastic Systems Group at MIT, Cambridge, MA.

Anima received the 2008 IEEE Signal Processing Society (SPS) Young Author award for her paper co-authored with Lang Tong appearing in the IEEE Transactions on Signal Processing. She is the recipient of the Fran Allen IBM Ph.D fellowship for the year 2008-09, presented annually to one female $\mathrm{Ph} . \mathrm{D}$. student in conjunction with the IBM Ph.D. Fellowship Award. She was named a finalist for the Google Anita-Borg Scholarship 2007-08. She received the Student Paper Award at the 2006 International Conference on Acoustic, Speech and Signal Processing (ICASSP). Her research interests are in the area of statistical-signal processing, information theory and networking. Specifically, she has been worked on distributed inference and learning of graphical models, routing and random-access schemes, error exponents and queueing models. She has served as a reviewer for IEEE Transactions on Signal Processing, IEEE Transactions on Information Theory, IEEE Transactions on Wireless Communications and IEEE Signal Processing Letters.

Joseph E. Yukich received the B.A. and Ph.D. degrees in mathematics from Oberlin College (1978)

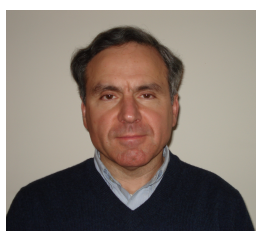
and the Massachusetts Institute of Technology (1983), respectively. He is the recipient of two Fulbright awards to France and has held visiting positions in Australia, Bell Labs, Cornell and Switzerland. He is currently a member of the faculty at Lehigh University.

He has research interests in spatial point processes, stochastic geometry, combinatorial optimization, and random networks and has authored the monograph Probability Theory of Classical Euclidean Optimization Problems, (Lecture Notes in Mathematics, volume 1675). His research has been supported by the National Science Foundation and the National Security Agency.

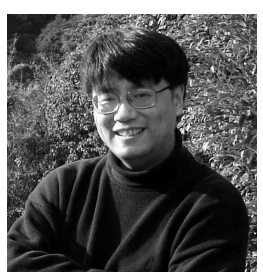

Lang Tong (S'87,M'91,SM'01,F'05) is the Irwin and Joan Jacobs Professor in Engineering at Cornell University Ithaca, New York. Prior to joining Cornell University, he was on faculty at the West Virginia University and the University of Connecticut. He was also the 2001 Cor Wit Visiting Professor at the Delft University of Technology. Lang Tong received the B.E. degree from Tsinghua University, Beijing, China, in 1985, and M.S. and Ph.D. degrees in electrical engineering in 1987 and 1991, respectively, from the University of Notre Dame, Notre Dame, Indiana. He was a Postdoctoral Research Affiliate at the Information Systems Laboratory, Stanford University in 1991.

Lang Tong is a Fellow of IEEE. He received the 1993 Outstanding Young Author Award from the IEEE Circuits and Systems Society, the 2004 best paper award (with Min Dong) from IEEE Signal Processing Society, and the 2004 Leonard G. Abraham Prize Paper Award from the IEEE Communications Society (with Parvathinathan Venkitasubramaniam and Srihari Adireddy). $\mathrm{He}$ is also a coauthor of five student paper awards. He received Young Investigator Award from the Office of Naval Research.

Lang Tong's research is in the general area of statistical signal processing, wireless communications and networking, and information theory. He has served as an Associate Editor for the IEEE Transactions on Signal Processing, the IEEE Transactions on Information Theory, and IEEE Signal Processing Letters.

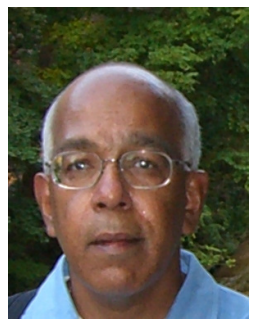

Ananthram Swami received the B.Tech. degree from IIT-Bombay; the M.S. degree from Rice University, and the Ph.D. degree from the University of Southern California (USC), all in Electrical Engineering. He has held positions with Unocal Corporation, USC, CS-3 and Malgudi Systems. He was a Statistical Consultant to the California Lottery, developed a Matlab-based toolbox for non-Gaussian signal processing, and has held visiting faculty positions at INP, Toulouse. He is with the US Army Research Laboratory (ARL) where his work is in the broad areas of signal processing, wireless communications, sensor and mobile ad hoc networks. He is an ARL Fellow.

Dr. Swami is a member of the IEEE Signal Processing Society's (SPS) Technical Committee (TC) on Sensor Array \& Multi-channel systems, a member of the IEEE SPS Board of Governors, and serves on the Senior Editorial Board of IEEE Journal of Selected Topics in Signal Processing. $\mathrm{He}$ has served as: chair of the IEEE SPS TC on Signal Processing for Communications; member of the SPS Statistical Signal and Array Processing TC; associate editor of the IEEE Transactions on Wireless Communications, IEEE Signal Processing Letters, IEEE Transactions on Circuits \& SystemsII, IEEE Signal Processing Magazine, and IEEE Transactions on Signal Processing. He was guest editor of a 2004 Special Issue (SI) of the IEEE Signal Processing Magazine (SPM) on 'Signal Processing for Networking', a 2006 SPM SI on 'Distributed signal processing in sensor networks', a 2006 EURASIP JASP SI on Reliable Communications over Rapidly Time-Varying Channels', a 2007 EURASIP JWCN SI on 'Wireless mobile ad hoc networks', and the Lead Editor for a 2008 IEEE JSTSP SI on "Signal Processing and Networking for Dynamic Spectrum Access". He is a co-editor of the $2007 \mathrm{Wi}$ ley book "Wireless Sensor Networks: Signal Processing \& Communications Perspectives". He has co-organized and co-chaired three IEEE SPS Workshops (HOS'93, SSAP'96, SPAWC'10) and a 1999 ASA/IMA Workshop on HeavyTailed Phenomena. He has co-led tutorials on 'Networking Cognitive Radios for Dynamic Spectrum Access" at ICASSP 2008, DySpan 2008 and MILCOM 2008. 